\title{
Agents in Object-Oriented Software Engineering
}

\author{
Alessandro F. Garcia ${ }^{1} \quad$ Carlos J. P. de Lucena $^{1} \quad$ Donald D. Cowan $^{2}$ \\ ${ }^{1}$ Software Engineering Laboratory - SoC+Agents/TecComm Group \\ Computer Science Department \\ Pontifical Catholic University of Rio de Janeiro - PUC-Rio \\ Rua Marquês de São Vicente, 225 - 22453-900 \\ Rio de Janeiro - Brazil \\ Phone/FAX: +55 (21) 512-2299 \\ e-mail:\{afgarcia,lucena\}@inf.puc-rio.br \\ ${ }^{2}$ Computer Systems Group \\ School of Computer Science \\ University of Waterloo \\ Waterloo, Ontario, N2L 3 G1 Canada \\ Phone/FAX: 519-888-4690/519-746-5422 \\ e-mail:dcowan@csg.uwaterloo.ca
}




\begin{abstract}
Software engineers of multi-agent systems (MASs) are faced with different concerns such as autonomy, adaptation, interaction, collaboration, learning, and mobility, which are essentially different from classical concerns addressed in object-oriented software engineering. MAS developers however have relied mostly on object-oriented design techniques and programming languages, such as Java. It often leads to a poor separation of MAS concerns and in turn to the production of MASs that are difficult to maintain and reuse. This paper discusses software engineering approaches for MASs, and presents a new method for integrating agents into object-oriented software engineering from an early stage of design. The proposed approach encourages the separate handling of MAS concerns, and provides a disciplined scheme for their composition. Our proposal explores the benefits of aspect-oriented software development for the incorporation of agents into object-oriented systems. We also illustrate our aspect-oriented approach through the Portalware multi-agent system, a web-based environment for the development of e-commerce portals.
\end{abstract}

Keywords: Multi-agent systems, software agents, software engineering, object-oriented systems, aspect-oriented software development.

\title{
1. Introduction
}

Agent technology has been revisited as a complementary approach to the object paradigm and as a means of designing and implementing complex distributed software. Agents and objects are abstractions recognizably different from a software engineering viewpoint $[1,2,3,12]$. Software engineers who design and implement multi-agent systems (MASs) are faced with concerns, such as agent autonomy, interaction, and adaptation that are not naturally supported by abstractions associated with object-oriented software engineering [1,3]. However, MAS developers however have relied mostly on object-oriented (OO) design techniques and programming languages, such as Java. It turns out that several MAS concerns are lost across the system objects [4]. It may lead to a poor separation of MAS concerns and in turn to the production of MASs that are difficult to maintain and reuse. Separation of concerns [5] is a well-known principle in software engineering used to achieve improved reusability and maintainability of complex software. With MASs growing in size and complexity, the promotion of separation and composition of concerns throughout the design and implementation process is critical to MAS engineers.

The importance of agent properties in today's software systems is reflected by the support provided for such properties in object-oriented middleware, programming languages, and implementation frameworks. In practice, the 
concept of object has been considered the basic abstraction for designing and implementing MASs. In fact, the notion of objects has been enriched with MAS properties naturally leading to the inclusion of agents into software systems. However, software engineers have largely relied on their experience and intuition in order to develop MASs based on object-oriented abstractions. This observation provides the rationale for investigating how to integrate agents neatly within object-oriented software engineering $[6,7]$. Agent-based software engineering has been studied from different perspectives, including agent-oriented methodologies for higher-level development phases [8, 9], conceptual modeling $[10,11,12]$, and implementation frameworks $[13,14,15]$. Implementation frameworks provide objectoriented APIs for MAS development, without providing guidelines for structuring agency concerns that are application dependent. In addition, most proposed methodologies are too high level and do not indicate how to master the complexity of these concerns based on abstractions from the object paradigm. Little work has been reported so far on the definition of a development method to structure agency concerns in software systems. As a consequence, MASs have been designed and implemented in an ad hoc way, and consequently agency concerns are lost across the object-oriented design models and code, making comprehension, reuse and maintenance difficult.

In this context, we propose an aspect-oriented method for MAS development that assists software engineers in introducing agents into object-oriented systems. Aspect-oriented software development [16, 17] is growing in popularity as the means of providing improved separation of concerns in design and implementation. It encourages modular descriptions of software systems by providing support for cleanly separating the object functionality from its crosscutting concerns. Aspect [16] is the abstraction used to modularize concerns that crosscut several system objects. Since a single agent is an abstraction much richer than an object, our approach is centered on the notion of aspects to associate system objects with agent properties in a transparent way. Objects encapsulate the basic functionality of the agents, while aspects are applied to modularize agency concerns and achieve improved separation of MAS concerns. Software engineers can then reason about agent behavior from different perspectives, and consider agency concerns in a stepwise fashion. Our proposal provides agent system developers with guidelines to reuse and evolve these aspects separately in order to compose the different agent aspects of an application.

Our method is independent of MAS implementation frameworks, such as JADE [18], ZEUS [19], and Retsina [20], and specific communication languages [21, 22]. We will illustrate the application of our method by introducing multiple software agents in Portalware [23], a web-based environment for the development of ecommerce portals. To implement this system, we have used AspectJ [24], which is an aspect-oriented extension to the Java programming language [25]. The empirical validation of our proposal is also presented based on qualitative and quantitative studies that we have conducted previously [26, 27]. 
The remainder of this paper is organized as follows. Section 2 gives a brief description of definitions of multi-agent systems, and introduces an example which is used to illustrate our approach. This section also presents the requirements for agency concerns which are not covered by object-oriented abstractions as based on the example. Section 3 overviews software engineering approaches for MASs in order to put our contributions into perspective. Section 4 introduces aspect-oriented software development and AspectJ. Section 5 presents our aspect-based approach for developing MASs, and applies it to the Portalware system. Section 6 describes some implementation issues. Section 7 presents the empirical validation of the proposed method, discussing the relative advantages and disadvantages of applying our approach. Finally, Section 8 discusses related work, and Section 9 presents some concluding remarks and directions for future work.

\section{Multi-Agent Systems: Definitions and Case Study}

As objects, agents encapsulate state and behavior, and provide services to their counterparts. Agents are commonly seen as an extension of objects [12, 28, 29, 30]. However, agents often are viewed as complex objects with an attitude [30], in the sense of objects with additional characteristics. As a result, development of MASs involves concerns supported by abstractions from the object paradigm and other concerns not directly supported. We term both kinds of concerns as agency concerns. In the following, we describe the main concerns software engineers face in the development of MASs. Sections 2.2 and 2.3 present our example and introduce the limitations of the object-oriented abstractions to capture agency concerns.

\subsection{Agency Concerns}

In general, the state of an agent is formalized by knowledge and is expressed by mental components. There are different proposals for representing agent knowledge, but mental components commonly used are beliefs, goals, actions and plans $[28,31,32,33]$. The behavior of an agent is respectively affected by and composed of agency properties. Agency properties are behavioral characteristics that an agent can have to achieve its goals. Table 1 summarizes the definitions for the main agency properties. These definitions are based on previous studies [12, 14, 28, 34] and our experience in developing MASs [23, 35, 36]. Agency properties can be classified in agenthood properties and additional properties. Autonomy, interaction and adaptation are considered in this work as fundamental properties of software agents, while collaboration, learning, and mobility are neither necessary nor sufficient conditions for agenthood [28] (see Table 1). However, note that this classification is only one of several possible ones. 


\begin{tabular}{|c|c|c|}
\hline & AGENCY PROPERTY & DEFINITION \\
\hline \multirow{3}{*}{ 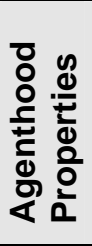 } & Interaction & $\begin{array}{l}\text { An agent communicates with the environment and other agents by } \\
\text { means of sensors and effectors }\end{array}$ \\
\hline & Adaptation & $\begin{array}{l}\text { An agent adapts/modifies its mental state according to messages } \\
\text { received from the environment }\end{array}$ \\
\hline & Autonomy & $\begin{array}{l}\text { An agent is capable of acting without direct external intervention; it } \\
\text { has its own control thread and can accept or refuse a request message }\end{array}$ \\
\hline \multirow{3}{*}{ 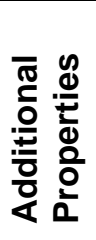 } & Learning & $\begin{array}{l}\text { An agent can learn based on previous experience while reacting and } \\
\text { interacting with its environment }\end{array}$ \\
\hline & Mobility & $\begin{array}{l}\text { An agent is able to transport itself from one environment in a network } \\
\text { to another }\end{array}$ \\
\hline & Collaboration & $\begin{array}{l}\text { An agent can cooperate with other agents in order to achieve its goals } \\
\text { and the system's goals }\end{array}$ \\
\hline
\end{tabular}

Table 1. An Overview of Agency Properties

In fact, in spite of the growing interest in MASs, there is no agreement on what actually constitutes agenthood, that is, what are the fundamental characteristics of agents. There is no agenthood definition widely accepted and it varies from researcher to researcher. In this paper, we consider an agent as an autonomous entity that encapsulates a state, interacts with its environment, and adapts itself based on successive interactions. A software agent is not usually found completely alone in an application, but often forms an organization with other agents. A MAS generally has several types of software agents [34], such as information agents, user agents, and interface agents. Each agent type incorporates the agenthood features, but provides different services. An agent type is characterized by the set of services it provides to its environment and other agent types. It is also associated with different agency properties in order to provide services and achieve its goals.

An agent can collaborate with other agents in order to use the counterpart services via some communication language $[21,22]$. Collaborative agents play different roles in pursuing their individual goals and work together with other agents in multiple contexts. Roles are application-dependent and are specific for each context. In order to cooperate, a plan is instantiated, and each agent plays a different role.

The agency properties are not orthogonal - in general, they interact with each other. For instance, the adaptation depends on autonomy since it is necessary to adapt the agent's state and behavior when the agent's autonomy capabilities determine whether to accept an incoming message. In addition, some agency properties are overlapping, such as interaction and collaboration. Collaboration is viewed as a more sophisticated interaction form, since the former comprises communication and coordination. Interaction is only concerned with communication, i.e. sending and receiving messages. During inter-agent collaboration, messages are also received from and sent to the participating agents. However, the collaboration property also defines how to collaborate, it addresses the 
coordination protocol. A simple coordination protocol consists of synchronizing with the agent waiting for a response.

\subsection{Software Agents in Portalware: A Case Study}

This section introduces a MAS in order to illustrate the application of agents, and the feasibility of our approach presented in Section 5. This system is derived from a case study undertaken in the SoC+Agents Group [36] at PUCRio in Brazil (from herein referred to as Portalware [23]). Portalware is a web-based environment that supports the development and management of e-commerce portals. This environment introduces a systematic approach for producing and maintaining portals by means of a clear separation of user roles. Portalware also supports communication facilities for coordinating and organizing the activities of the portal developers. Software agents have been introduced to Portalware in order to assist its users with time-consuming activities and automate repetitive user tasks. We selected this system as a case study, because the agency concerns handled in this project are ones typical of many existing applications. This MAS encompasses several agency concerns, including agent types, roles, collaboration, interaction, adaptation, and autonomy.

Different roles are attributed to each Portalware user, but the main ones are: (i) content supplier, (ii) reviewer, and (iii) editor. Content supplier (CS) is responsible for providing the portal with information (for instance, news). A reviewer examines each of the content segments, and may change them if this is thought desirable or necessary. The editor is responsible for selecting from the available content segments for publishing and for making individual agents reviewers or content suppliers. Each Portalware user can be assigned one or more roles.

Portalware encompasses three agent types: (i) interface agents, (ii) information agents, and (iii) user agents. Each agent type provides different services, but everyone implements the fundamental characteristics defined by agenthood. Each agent type has different application-specific agency properties. Figure 1 presents the agent types in the Portalware environment, and the distinct agency properties associated with them. Interface agents monitor the graphical interface in order to interact with Portalware users. They learn short-cuts by capturing preferences, and by receiving explicit instructions from the user. For instance, interface agents operate while a content supplier (CS) is authoring content segments, using keywords entered in the document and its acquired model of the user's preferences. Interface agents do not have collaborative capabilities since they do not cooperate with other software agents. User agents represent Portalware users and are implemented to reduce the need for cross-talk between working users. Since editors, reviewers, and CSs need to communicate with each other to maintain the web portal, user agents incorporate these roles and collaborative capabilities for automating and supporting collaboration in different contexts. The agent representing an editor has the responsibility to contact the prosprective reviewers and CSs and negotiate with them for the use of their services. 


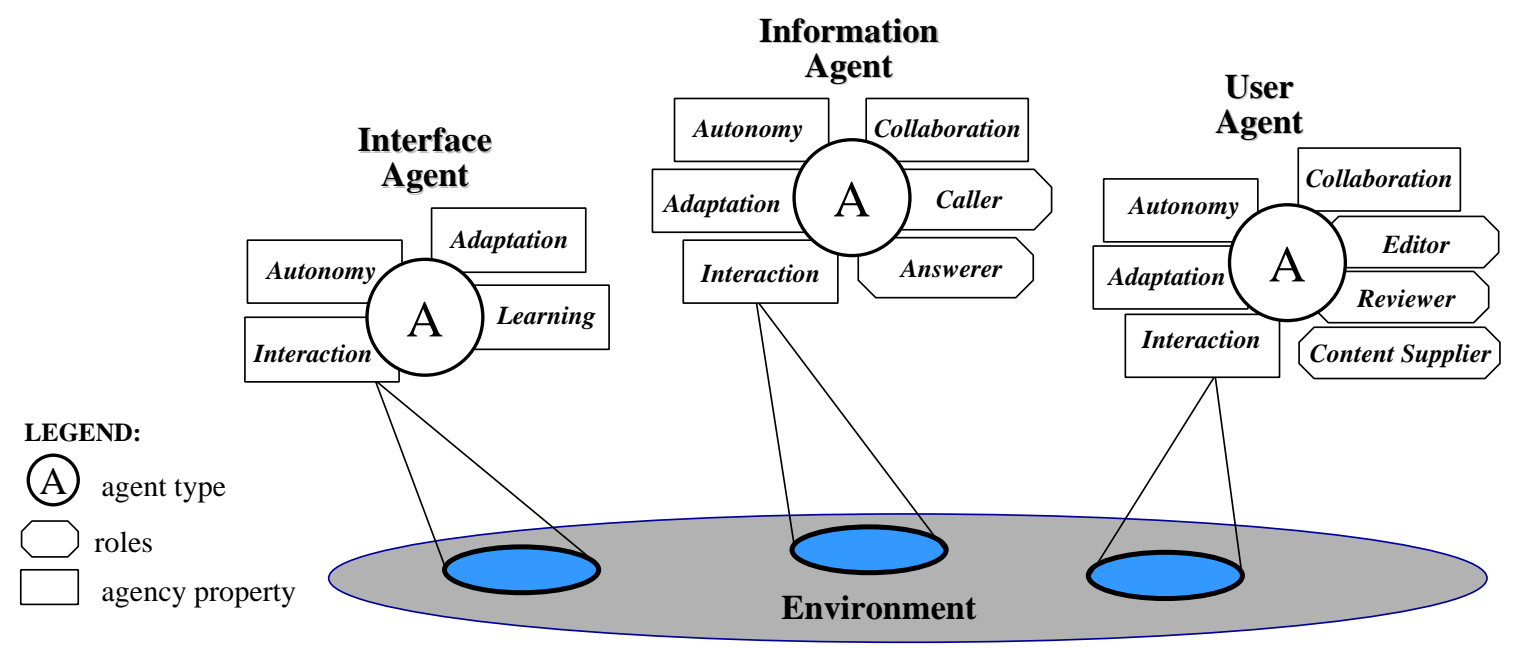

Figure 1. Portalware Agents.

Portalware users often need to search for information which is stored in two different databases. Each information agent is attached to a database, and contains plans for searching for information. The search plan determines the agent's searching service. An alternative collaborative plan is used when an information agent is not able to find the information in the attached database. This plan plays the caller role in order to call the other information agents and ask for this information. Similarly, the latter activates a collaborative plan that plays its answerer role so that it can receive the request and send the search result. Note that both of them may play the caller and answerer roles.

\subsection{Object-Oriented Development of Portalware}

The agent-oriented modeling of Portalware was based on Elammari's modeling language [8] and on the TAO conceptual framework [12]. TAO was used because it elicits common abstractions used in MAS analysis and design. Based on these agent-oriented concepts, Portalware was designed and implemented with abstractions from objectoriented software engineering. UML notations [37] and the Java language were respectively used to generate the object-oriented designs and implementation. Figures 2 represents a slice of the object-oriented design for Portalware, and Figure 3 presents some lines of Java code. The object-oriented design has emphasized the use of design patterns [38] in order to produce an extensible and modifiable system. For example, we used the Role Pattern [39] to represent the agent roles, and the Mediator pattern [38] to associate different agency properties with different agent types of Portalware. We have also used design patterns that are tailored to the agent domain $[14,40]$.

However, as the needs of the Portals market change rapidly the deficiencies in the software engineering techniques often used was becoming increasingly apparent to portal developers. To survive, Portalware should remain extensible and modifiable, and so its design and implementation should be able to respond to changes quickly. In the object-oriented development of Portalware, we detected several recurrent limitations in the development of other MASs $[35,36]$. These limitations were the origins of a number of intrusive changes and reuse difficulties as the system evolved. The first limitation is that object-oriented abstractions are not able to modularize agency concerns, 
such as collaboration. Figure 2 shows the problem related to the collaboration concern in the Portalware design. This concern crosscuts several system objects, making it difficult to understand the design and modify the system code. Any changes related to this concern are likely to involve many modules that implement the concern. Even worse, it was not possible to encapsulate an agency concern in a unique design pattern, as depicted in Figure 2, so that it could not be reused as a single design block.

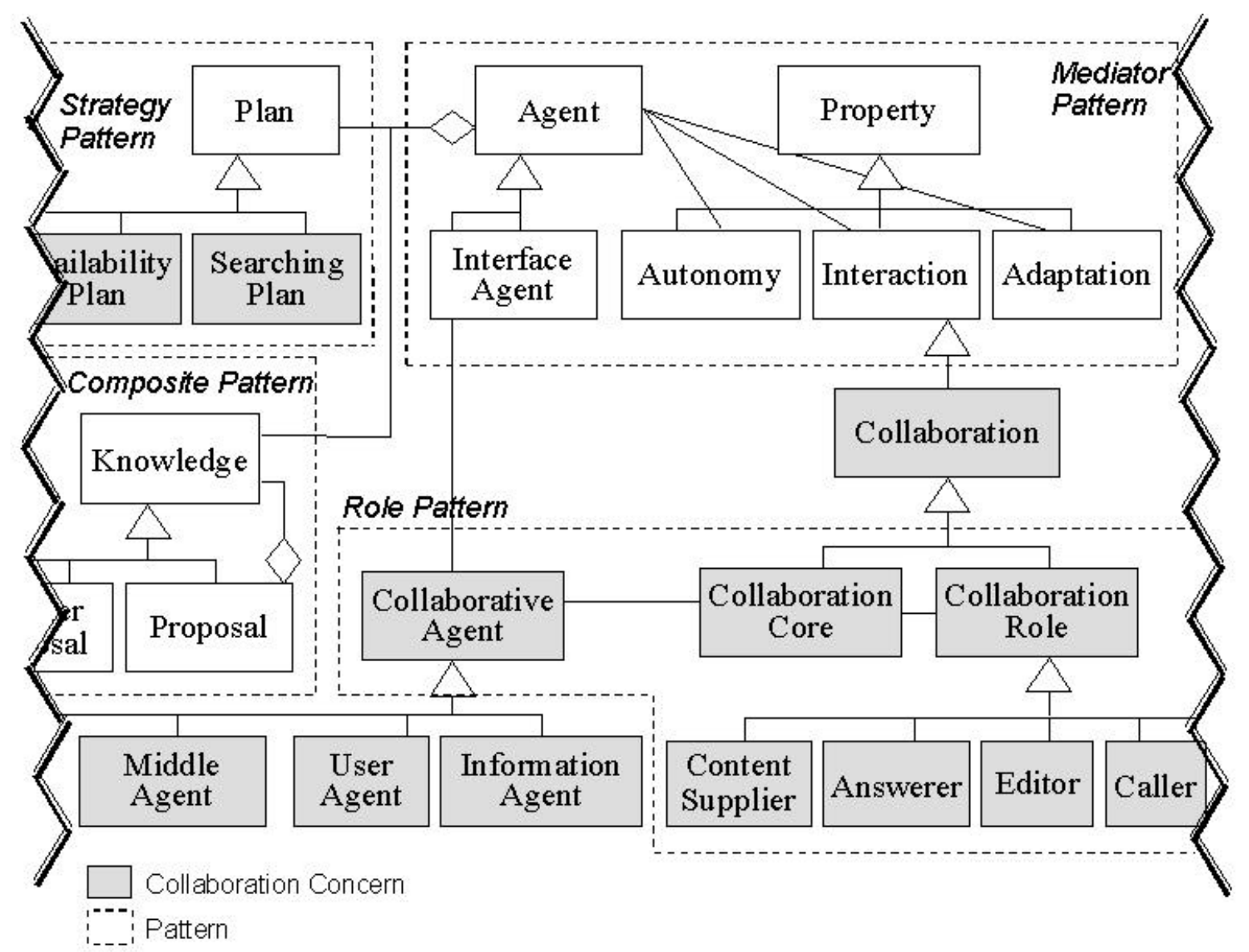

Figure 2. A Subset of the Oriented-Oriented Design for Portalware MAS

Another problem relates to the fact that it is not easy to understand the agency concerns that are attached to a specific agent type. For example, it is not clear in the design that a user agent is associated with the CS, reviewer, and editor roles (Figure 2). The use of the Role pattern helps to modularize the roles, but it increases the number of classes in the system, which are created to control the composition of role instances and agent instances. This in turn resulted in several misalignments between software artifacts generated in higher-level development phases and the ones described in the object-oriented design. Since an agent is intended to participate in a number of heterogeneous collaborations, roles should be introduced to the agent's core behavior in a way that is structured and non-intrusive. 


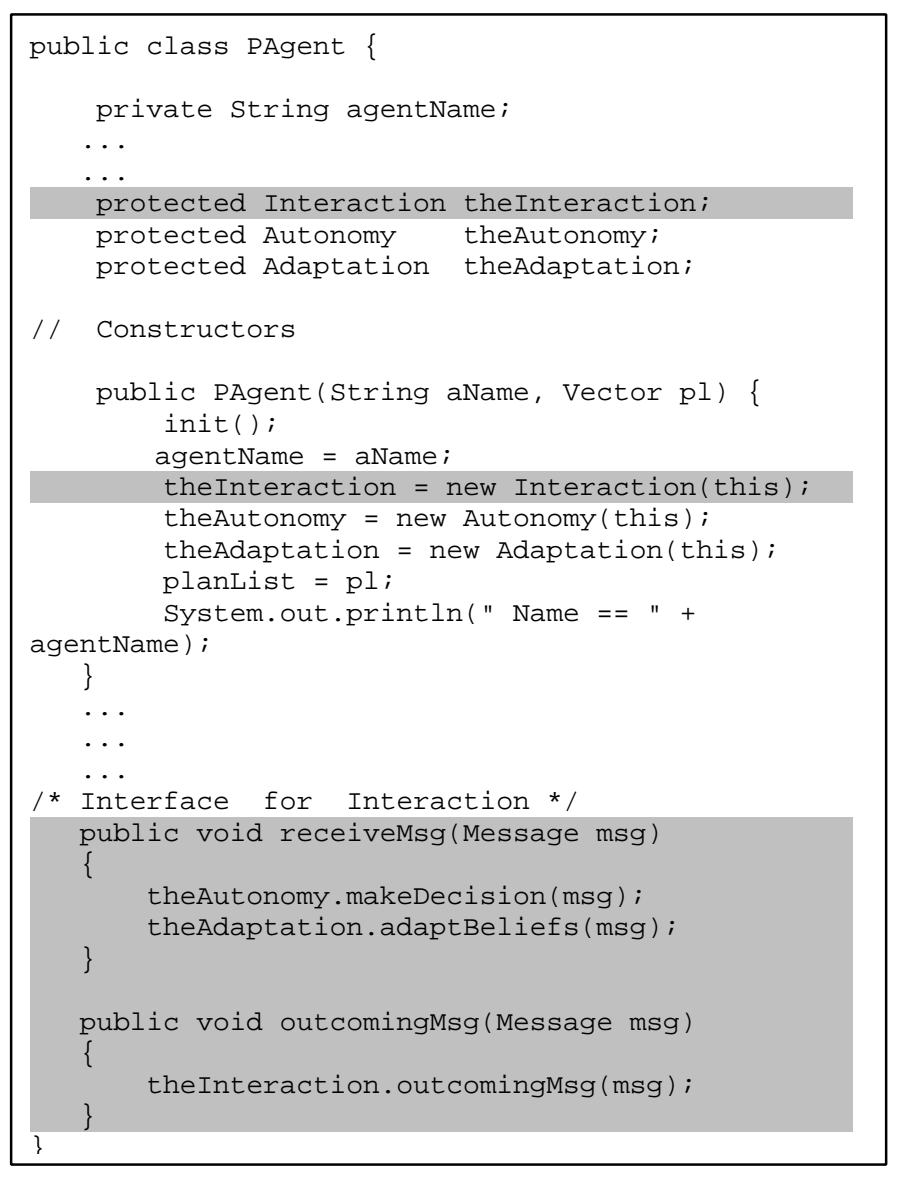

Figure 3. Java Code of Portalware

The problem was not restricted to the system design. The handling of MAS concerns was even more difficult from the implementation viewpoint, since most agency concerns intrude into the basics system functionality. There were agent properties, such as adaptation and autonomy, that should be modularized, but their implementation was instead diffused throughout the methods representing the agent services. Figure 3 illustrates this problem for the Agent class. The lack of support for handling the interactive and overlapping nature of agency properties (Section 2.1) also limited the understanding, maintainability and reusability of MASs. Note, for example, the body of the method receiveMsg(...) (interactive behavior of the agent) is amalgamated with explicit calls to: (i) the makeDecision(...) method which implements the autonomous behavior of the agent, and (ii) the adaptBeliefs(...) method which implements the adaptive behavior of the agent. In short, Portalware programmers faced lack of support for explicitly isolating and composing agency concerns based on object-oriented abstractions available in the Java language. The inherent complexity in MAS construction requires the application of appropriate software engineering approaches to promote separation of the concerns. Different existing approaches for MAS engineering are discussed in the next section in terms of their support for separation of concerns. This section also puts our approach into perspective. 


\section{Software Engineering for Multi-Agent Systems}

Separation of concerns is a well-established software engineering principle that uses high-level abstractions to hide complexity [5]. An agency concern can be modularized throughout the software lifecycle using three different categories of abstractions: modular abstractions, aspect abstractions, and architectural abstractions. Figure 4 provides a framework that identifies existing abstractions in these categories. Modular abstractions are useful to encapsulate simple and orthogonal concerns on modules, such as data, functions, classes, components, and agents. Modular abstractions are supported by modeling languages, programming languages, and implementation frameworks. However, complex concerns are not able to be simply captured by modular abstractions. These abstractions only provide limited support for separation of concerns [16].

The aspect abstractions are aimed at encapsulating concerns that naturally crosscut several system modules defined during the software lifecycle. There are different aspect abstractions available in the literature, such as aspects [16], viewpoints [41], features [42], and subjects [43]. Each of these abstractions is tailored to different development phases. Aspects are supported by design and programming languages (Section 4). Finally, architectural abstractions are able to represent system concerns according to different perspectives, such as structural perspectives and behavioral perspectives. These abstractions are usually provided by diagrams in modeling languages, such as class diagrams and sequence diagrams, and generally are not present in the code.

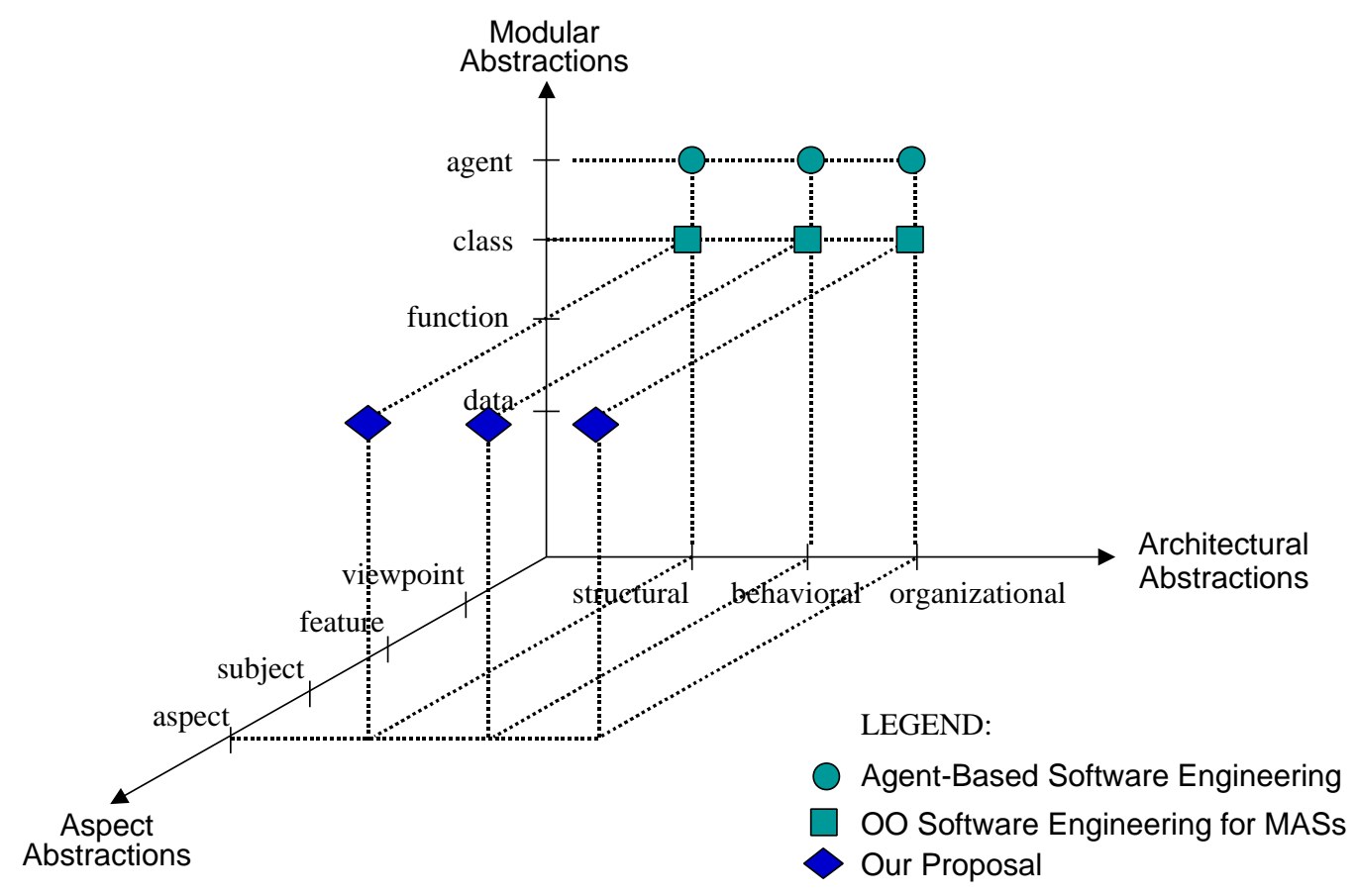

Figure 4. Software Engineering Approaches for MASs.

According to the framework pictured in Figure 4, we classify current approaches to multi-agent software engineering into two categories: (i) agent-based software engineering, and (ii) object-oriented software engineering 
for MASs. Although these approaches can be viewed as complementary to some extent, the central abstraction is the agent in the first one while the object is the key abstraction in the second one. Both approaches have focused on modular decomposition and architectural decomposition. Our proposal follows the second approach and extends it with the application of aspects as an abstraction to improve separation of MAS concerns. The following subsections briefly discuss the current research in agent-based software engineering and object-oriented software engineering for MASs, respectively. Section 4 introduces the aspect notion and outlines aspect-oriented software development.

\subsection{Agent-Based Software Engineering}

Researchers in agent-based software engineering (ABSE) such as those in [1] argue persuasively that adopting a multi-agent approach to system development affords software engineers a number of significant advantages over contemporary methods and, therefore, they propose a new software engineering. According to these researchers, multi-agent systems are often more complex than object-oriented systems and hence object-oriented abstractions generally fail to provide support for agency concerns. In this approach, agents are a new modular abstraction that substitutes for object abstraction, providing the foundation for an essentially different paradigm. As a result, proponents of this approach claim it is necessary to develop new software engineering techniques and methods that are specifically tailored to agents, as well as software architectures, programming languages and tools supporting these techniques, methods and methodologies.

Up to now, ABSE researchers have dedicated special attention to the development of conceptual frameworks [11, 12], and high-level modeling languages and methods [8, 9]. For example, GAIA [9] is an agent-oriented development approach composed of a set of modeling languages and a method to use these agent-oriented languages. However, it is oriented towards high-level development phases such as analysis and design. It does not provide support for MAS developers to integrate agents neatly in object-oriented design and implementation. In addition, it supports architectural and modular abstractions, such as agents and goals. It does not support the aspect abstractions. TAO [12] is an agent-centric conceptual framework that elicits a set of abstractions to agent-based software engineering. TAO specifies the relationships between agent-oriented abstractions and object-oriented abstractions, but it also does not support aspect abstractions. Moreover it is also dedicated to early development phases and does not encompass a development method for detailed design and programming. The References 44, 45, and 46 give a more complete survey on agent-oriented languages, methodologies, and conceptual frameworks.

\subsection{Object-Oriented Software Engineering for MASs}

In contrast to the ABSE approach, other researchers $[3,7,14,15,28]$ propose the integration of agents into the object-oriented world and thus, they think of objects and agents as complementary abstractions. As a result, they have concentrated on extending existing techniques from object-oriented software engineering to agent-based systems. The 
central idea of this approach is the addition of new features to objects so they become agents. This research approach is based on the fact that object-oriented software engineering has proved to be extremely powerful for building complex systems, which promote modularity, maintainability, and reusability. Moreover, object-oriented software engineering has evolved by incorporating successful techniques, such as object-oriented frameworks [47] and design patterns [38]. As a consequence, this approach's proponents believe that software engineering approaches for MASs should be defined in the light of object-orientation.

This approach's researchers have worked on the definition of object-oriented frameworks for MAS development, such as JADE [18], ZEUS [19], and RETSINA [20]. For example, JADE is an object-oriented framework to develop agent applications in compliance with FIPA specifications [48] for interoperable MASs. The goal is to simplify development while ensuring standard compliance through a comprehensive set of system services and agents. JADE is considered an agent middleware that implements an agent platform and a development framework. JADE provides a standard API on which MASs engineers can rely. The JADE interfaces provide the ability to manage basically three entities: the agent platform, individual agents, and agent organizations. However, JADE and other FIPA-compliant agent implementations deal with the system functionality that is not peculiar of the agent internals and that are independent of the applications, such as message transport, encoding and parsing, or agent lifecycle. Although the object-oriented frameworks are essential to MAS development, MAS developers are left without a development method to structure agency concerns (Section 2.1) based on object-oriented abstractions. As a result, implementation frameworks have different goals and the existing solution only support modular abstractions.

Moreover, like ABSE researchers, this approach's proponents have also dedicated attention to the development of high-level modeling languages [46, 49], where object is the central abstraction of software development. Nevertheless these proposals generally are oriented to modeling diagrams and do not provide a principled method for structuring agency concerns based on abstractions from object-oriented detailed design and implementation. To our knowledge, the only proposal that supports an object-oriented development method is the Kendall's work [14]. Her approach is based on an architectural pattern and a system of object-oriented design patterns. The layered agent architectural pattern separates the different agency concerns in terms of layers, such as sensory layer, collaboration layer, and so on. Each design pattern is viewed as a mini-method to structure each agency concern based on objects. However, the Kendall's method does not support aspect abstractions to encapsulate concerns that naturally crosscut objects of MASs (Section 2.3). Section 7 compares a pattern-oriented approach with our aspect-oriented proposal (Section 4). The next section introduces aspect-oriented software development which provides the foundations for our proposed method (Section 5). 


\section{Aspect-Oriented Software Development}

Aspect-oriented software development (AOSD) $[16,17]$ has been proposed as a paradigm for improving separation of concerns in software design and implementation. The central idea is that while modular abstractions of objectoriented languages are extremely useful, they are inherently unable to modularize all concerns of interest in complex systems. Aspect-oriented software development does for concerns that naturally cut across modules what objectoriented software engineering does for concerns that are naturally hierarchical - it provides abstractions that explicitly capture the crosscutting structure. Thus, the goal of AOSD is to support the developer in cleanly separating components (classes) and aspects (crosscutting concerns) from each other, by providing abstractions that make it possible to modularize and compose them to produce the overall system.

Aspects are defined as system properties that crosscut (i.e., cut across) classes in a system's design and implementation. Separating aspects from classes requires a mechanism for composing - or weaving - them later. Central to the process of composing aspects and components is the concept of join points, the elements of the component language semantics with which the aspect programs coordinate. A typical example of join point is method call. In the context of this work, aspects are a unified abstraction to design and implement agency properties. For example, a class is used to represent the basic services of an agent, and an aspect is used to modularize the adaptation property of that agent. This adaptation aspect is attached to the agent class using join points, such as calls to the methods implementing the basic services. In another words, the adaptation aspect crosscuts the agent class, and they are woven using the compiler (weaver) of an aspect-oriented language.

AspectJ [24] is an aspect-oriented language that extends the Java programming language [25]. AspectJ provides support for modular implementation of a range of crosscutting concerns. Join points are well-defined points in the dynamic execution of a program (Figure 5). Examples of join points in AspectJ are method calls, method executions, and field sets and reads. Pointcuts are collections of join points. Advice is a special method-like construct that can be attached to pointcuts. In this way, pointcuts are used in the definition of advices. There are different kinds of advice: (i) before advice runs when a join point is reached and before the computation proceeds, i.e. that runs when computation reaches the method call and before the actual method starts running; (ii) after advice runs after the computation "under the join point" finishes, i.e. after the method body has run, and just before control is returned to the caller; (iii) around advice runs when the join point is reached, and has explicit control whether the computation under the join point is allowed to run at all. Aspects are modular units of crosscutting implementation that are associated with one or more objects, comprised of pointcuts, advices, and introduction. Introduction is a construct that defines new ordinary Java member declarations to the object to which the aspect is attached (such as, attributes and methods). Weaver is the mechanism responsible for directing the normal control flow to an advice, when program 
execution point is at a join point (Figure 5). In the current version of AspectJ, almost all of the weaving process is realized as a pre-processing step at compile-time [24].

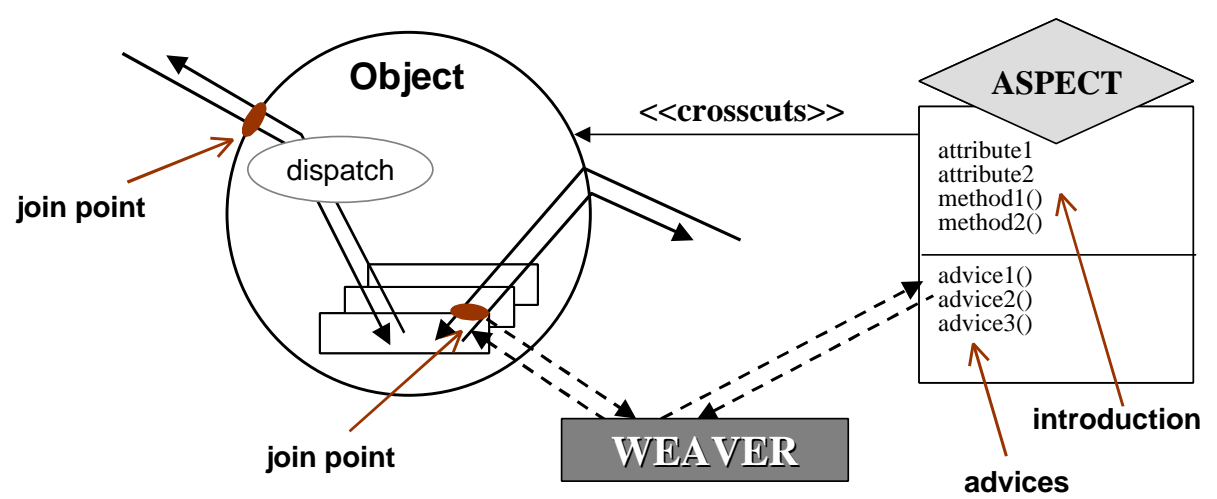

Figure 5. AspectJ Mechanisms

Although there are aspect-oriented programming languages that are general purpose (such as AspectJ), the most challenging problems in developing aspect-oriented software are the identification and structuring of the system aspects. Up to now, developers of aspect-oriented software have largely relied on their experience and intuition in order to capture the crosscutting concerns in the system, and how these concerns can be best organized in terms of the aspect abstractions. Even worse, there is no systematic procedure for designing the MASs, without offering specific insights into how to use the aspect abstractions to modularize agency concerns and produce MASs which are easy to understand, maintain, and reuse. The next section presents our proposal to cover this gap.

\section{An Aspect-Oriented Method}

The purpose of the method is to support software engineers in covering the most important agency concerns (Section 2.1). It is not be confused with a generic OO software framework (Section 3.2). In fact, our method is independent of MAS implementation frameworks, such as JADE, ZEUS, and Retsina, and specific communication languages, such as ACL [21] and KQML [22]. The perception of such independence is important for putting into perspective the scope of this work since we do not focus on details of implementation frameworks or communication languages. Our method aims to explore the benefits of aspect-oriented abstractions to structure a MAS, while leading to the construction of MASs which are easy to understand, maintain and reuse. The proposed method addresses the problems usually detected in the development of MASs based on the object paradigm (Section 2.3).

Our approach is based on the assumption that an agent is an object with added features and the aspect notion is used to enrich objects with agent properties in a transparent way. Objects can then be considered the basic structure for building agents. Object-oriented abstractions provide facilities to implement the basic structure and behavior of agents, and the inheritance concept enables specialization of agent types. However, since a single agent is a much richer abstraction than an object, our approach emphasizes separation of agency concerns in order to reason about the 
agent behavior from different perspectives. It considers the agency concerns, which are additional to object concerns in a stepwise fashion. Our method first guides the organization of the concerns that are more naturally supported by object-oriented abstractions, and later the concerns which are not. Our method describes a set of architectural decisions, which contribute to improve the maintainability of MASs and enable better reuse of agency concerns (Figure 6).

In particular, our aspect-oriented approach is structured in terms of the agency concerns (Section 2.1), namely: (i) agent state and behavior, (ii) agency properties and agenthood, (iii) agent types, (iv) additional agency properties, and (v) collaboration and roles. In addition, the method prescribes how to compose all agency concerns and how to evolve and reuse them throughout the software lifecycle. We adopt UML diagrams [37] as the modeling language throughout this paper. The design notation for aspects based on [59]: aspects are represented as diamonds, the first part of an aspect represents introductions, and the second represents pointcuts and their attached advices. Each advice is declared as follows: adviceKind(pointcut):adviceName. We have omitted most method parameters for simplicity purposes. 


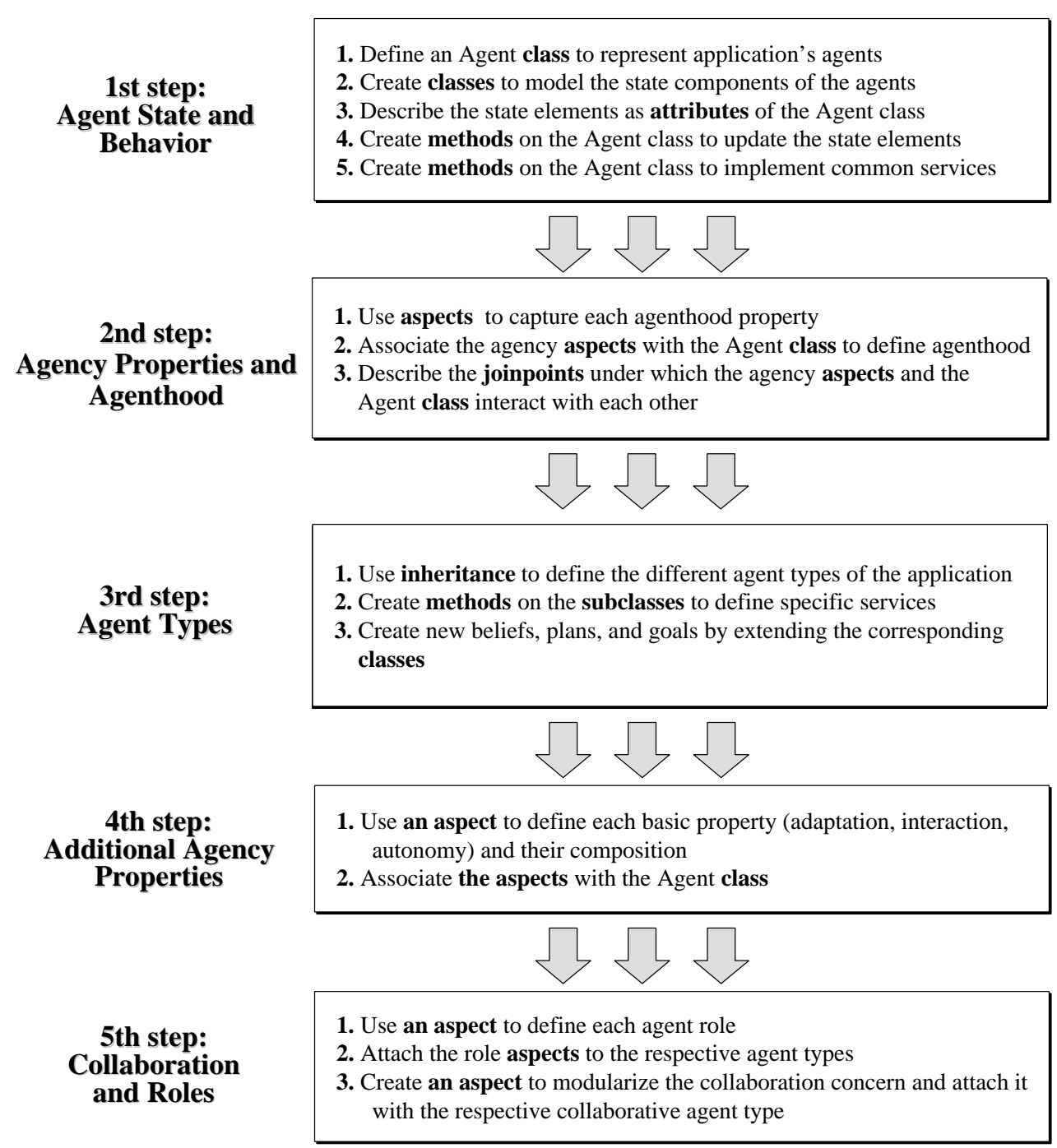

Figure 6. The Aspect-Oriented Method for MAS Development

\subsection{Agent State and Behavior}

In our approach, classes are used to represent agents and their constituent state components. This approach is supported by a number of OO implementation frameworks, such as JADE (Section 3.2). Classes represent agents as well as state components. The Agent class specifies the core state and behavior of an agent, and should be extended to create the application's agents. Each agent is attached to a name, which uniquely identifies the agent in the MAS. The services of the agents are implemented by the methods in the Agent subclasses, where each method represents a specific action. Methods of the Agent class are used to update its basic state and implement agent actions, which are common to all agent types in the application. Classes are also used to represent the internal state components of an agent. An agent has, among other attributes, collections of beliefs, goals and plans (Section 2.1). In practice, these are collections of Belief, Goal, and Plan objects and the Agent class provides standard operations for querying, inserting, updating and deleting an element. Figure 7 presents the main classes that represent the agent state and behavior. 
Beliefs represent the agent's observations in relation to its external environment. In other words, beliefs model the external environment with which an agent interacts. Changes in an agent's beliefs trigger the re-evaluation of the goals of the agent. Changes to the agent goals result in preempting some plans and initiating new plans. Execution of plans affects the environment, which in turn changes the beliefs, and so on. A goal may be realized through different actions and plans. A plan describes a strategy to achieve an internal goal of the agent, and the selection of plans is based on agent beliefs. In this way, the behavior of agents is driven by the execution of their plans that select actions in order to achieve the stated goals. Actions and plans are used to implement the agent services. Plans are associated with pre-conditions and post-conditions [8]. Pre-conditions list the beliefs that should be held in order for the plan to be executed, while post-conditions describe the effects of executing a succesful plan using an agent's beliefs.

The attributes of an Agent object should hold references to objects that represent these elements, namely Belief, Goal and Plan objects. Application designers must subclass the Belief, Goal and Plan classes to define their agents according to the application requirements. The Belief class should have the following attributes: (i) a name for identifying belief instances, (ii) a list of the goals that may be affected by changes to the belief, and (iii) dependentapplication attributes. The Belief class has methods for querying and updating a belief and relating goals with a belief.

The Goal class has a name, set and get methods, and the list of plans to accomplish the goal. This is because a goal may have different plans, and hence a Goal object may have more than one associated Plan object. A Goal object can be decomposed into subgoals. The Goal class also may include a utility value that indicates how valuable the goal is to a higher-level goal of the system. In addition, Goal has two abstract methods: changeBelief(...) and eval(...). The former is automatically invoked by a changed belief that affects the goal. It allows the goal to respond to belief changes. The latter can be used to compute the utility value. Its implementation is application dependent and can be, for example, a conventional decision tree, or any other evaluation mechanism appropriate for the type of agent. 


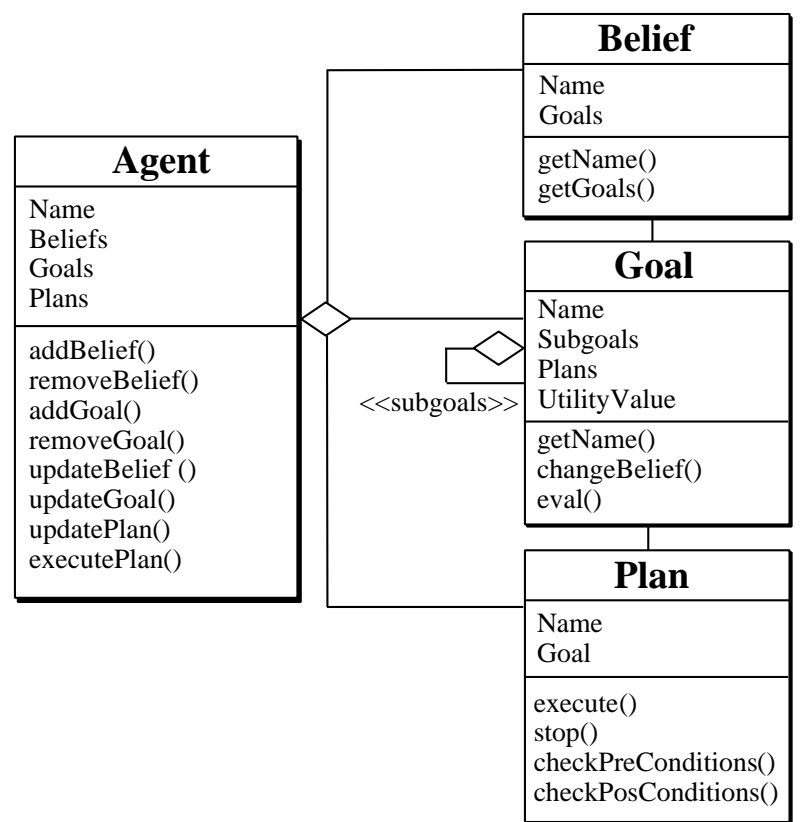

Figure 7. Agent State and Behavior.

The Plan class has an identifying name and an abstract execute() method which must be invoked to start a plan. This generic class may implement the Thread class. A subclass of Plan must implement the execute() method according to the concrete plan. The implementation may invoke KQML/FIPA performatives [21, 22] to communicate with other agents as well as perform conventional and knowledge based computations. In general, plans can be implemented as a sequence of actions, which are dynamically generated according to reasoning steps. Plan has a stop() method which can be invoked to terminate the plan. The Plan class also defines methods to check preconditions and set post-conditions (Section 2.1).

\subsection{Agency Properties and Agenthood}

Aspects should be used to implement the agency properties an agent incorporates. These aspects are termed agency aspects. Each agency aspect is responsible for providing the appropriate behavior for an agent's agency property. Figure 8 depicts the aspects, which define essential agency properties for agenthood: interaction, adaptation, and autonomy. These agency aspects affect both core states and behavior of agents (Section 2.1).

Interaction is the agency property that implements the communication with the external environment, i.e. the message reception and sending. An agent has sensors to receive messages, and effectors to send messages or act on the environment [14]. Since agents are autonomous software entities, the agent itself starts its control thread and decides between accepting and rejecting incoming messages. Since the message is accepted, the agent may have to adapt its mental state. The adaptation consists of processing an incoming message and defining which mental component is to be modified: beliefs can be updated, new goals can be set, and consequently plans can be selected. 


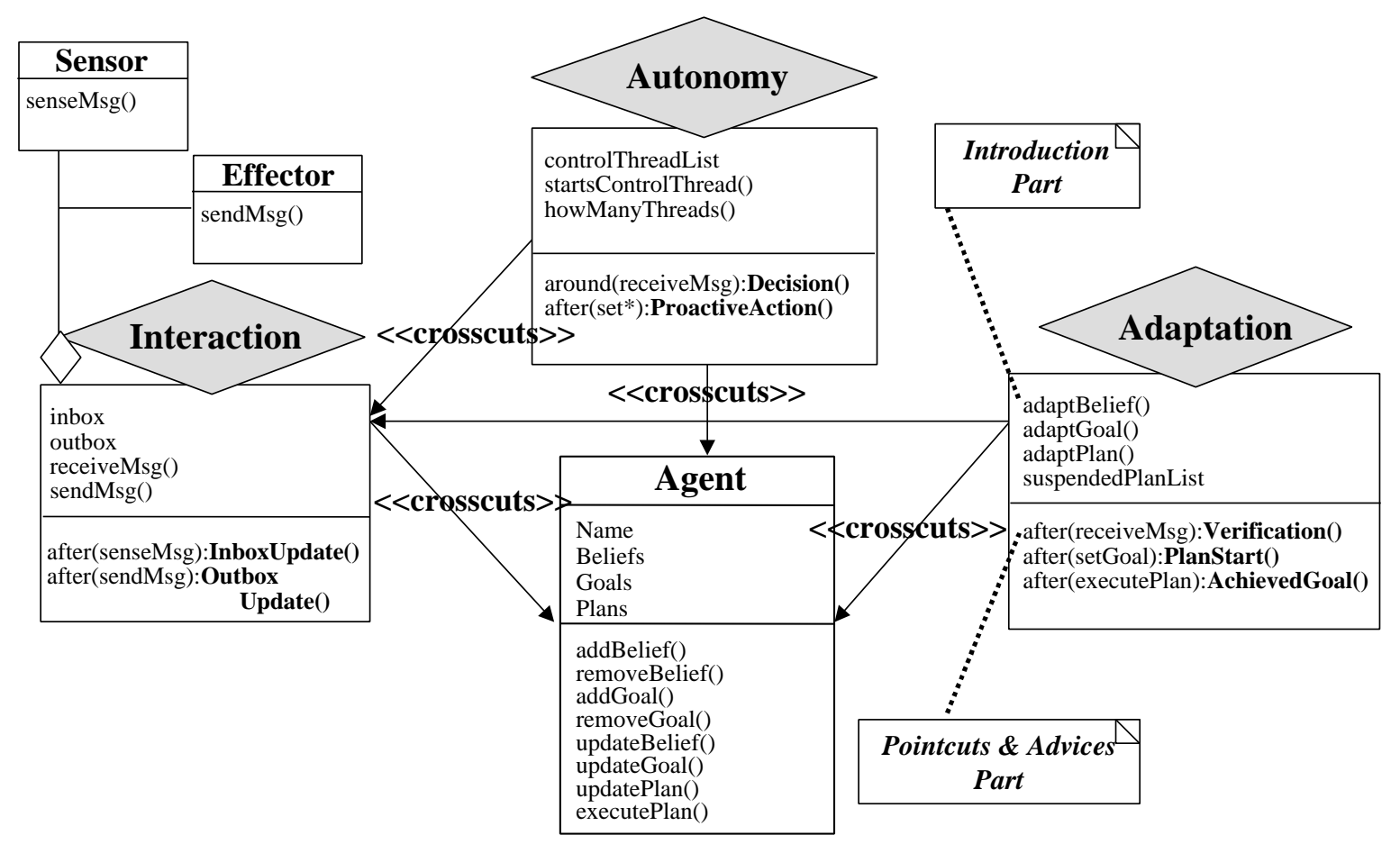

Figure 8. Agency Aspects and the Design for Agenthood.

For example, when the Interaction aspect is associated with the Agent class, it makes any Agent instance interactive. In other words, the Interaction aspect extends the Agent class's behavior to send and receive messages. This aspect updates messages and senses changes in the environment by means of sensors and effectors. The introduction part is used to add the new functionality related to the interaction property. The Sensor and Effector classes represent sensors and effectors respectively, and cooperate with domain-specific environment classes. When a message is received by means of a sensor, the Interaction aspect needs to update its inbox. So, the receptions of calls to the senseMsg() method are defined as a pointcut (Figure 8), and the InboxUpdate() after advice is associated with this pointcut. Similarly, the OutboxUpdate() after advice is attached to the putMsg() method in order to update the agent outbox. Since the process of sending and receiving messages occurs quite often in multi-agent systems and cuts across the agent services, the implementation of this process as an aspect is a design decision that avoids code duplication and improves reuse.

The Autonomy aspect makes an Agent object autonomous, it encapsulates and manages one or more independent threads of control, implements the acceptance or refusal of a service request and for acting without direct external intervention (Section 2.1). For example, the Decision() around advice implements the decision-making process by invoking specified decision plans when a message is received. Then, this advice is attached to the pointcut that is a collection of receptions of calls to the receiveMsg() method. The ProactiveAction() after advice implements the agent ability to act without direct external intervention (proactive behavior); to each invocation of methods with prefix set* (i.e., to each state change), this advice checks if a new plan must be started. 
The Adaptation aspect makes an Agent object adaptive, it adapts an agent's state (beliefs and goals) and behavior (plans) according to message receptions. As a consequence, this aspect crosscuts the Agent class and the Interaction aspect so that it is possible to perform state and behavior adaptations based on messages received from the environment by means of the receiveMsg() method. The Verification after advice verifies if state change is needed and which state component must be adapted. The AdaptBelief(), AdaptGoal() and AdaptPlan() methods themselves, defined in the introduction part, are responsible for updating beliefs, goals, and plans, respectively. The Adaptation aspect also implements the following behaviors: (i) adapts the agent behavior by starting appropriate plans when new goals are set (PlanStart() after advice), and (ii) adapts the agent's goal list by removing a goal when this goal is achieved, i.e. when the execution of the corresponding plan is finished successfully (AchievedGoal() after advice).

\subsection{Agent Types}

Our approach proposes the use of inheritance in order to create different agent types. Different types of agents are organized hierarchically as subclasses that inherit from the root Agent class. The methods of these subclasses implement the actions of each agent type. Figure 9 illustrates the subclasses representing the different kinds of agents of our case study (Section 2.2): (i) the InterfaceAgent class, (ii) the InformationAgent class, and (iii) the UserAgent class. For example, the method search(Keyword) of the InformationAgent class implements the services of information agents searching for information according to a specified keyword.

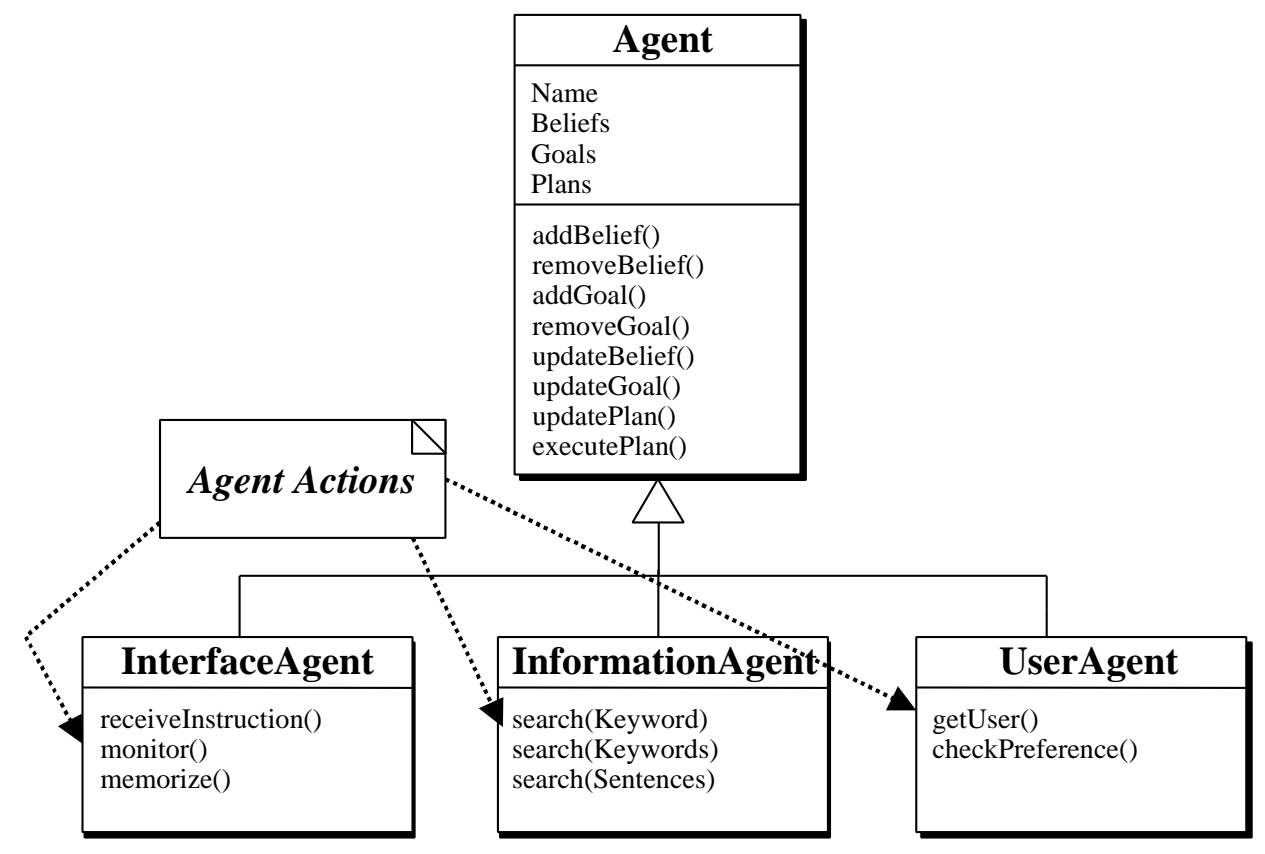

Figure 9. Agent Types. 


\subsection{Additional Agency Properties}

During the execution of plans, each agent type alternatively: (i) extends or refines its knowledge when interacting with its environment (learning), (ii) moves itself from one environment in a network to another (mobility), and (iii) joins a conversation channel with other agents (collaboration). Each agent type typically has different applicationspecific capabilities and agency properties.

The agency aspects that are specific to each agent type are associated with the corresponding subclasses (Figure 10). Note that the different types of software agents inherit the agency aspects attached to the Agent superclass. As a consequence, the three agent types reuse the agenthood features and only define their specific services and aspects. For example, the InformationAgent and UserAgent classes are associated with the Collaboration aspect, while the InterfaceAgent class is attached to the Learning aspect. The Collaboration aspect extends the Interaction aspect by implementing the synchronization of the agents participating in a collaboration (coordination protocol). It locks the agent sending a message as well as unlocks it when receiving the response. The Learning aspect introduces the behavior responsible for processing new information when the agent state is updated.

\subsection{Collaboration and Roles}

Aspects should be used to implement the roles of an agent. These aspects are termed role aspects. A role aspect is a part of an agent which defines the activity of the agent within a set of collaborations. As a result, it decouples the agent's basic services from the role's semantics, which in turn improves MAS understanding, reusability and evolution. Since an Agent object needs to include multiple roles, different roles aspects are associated with this object.

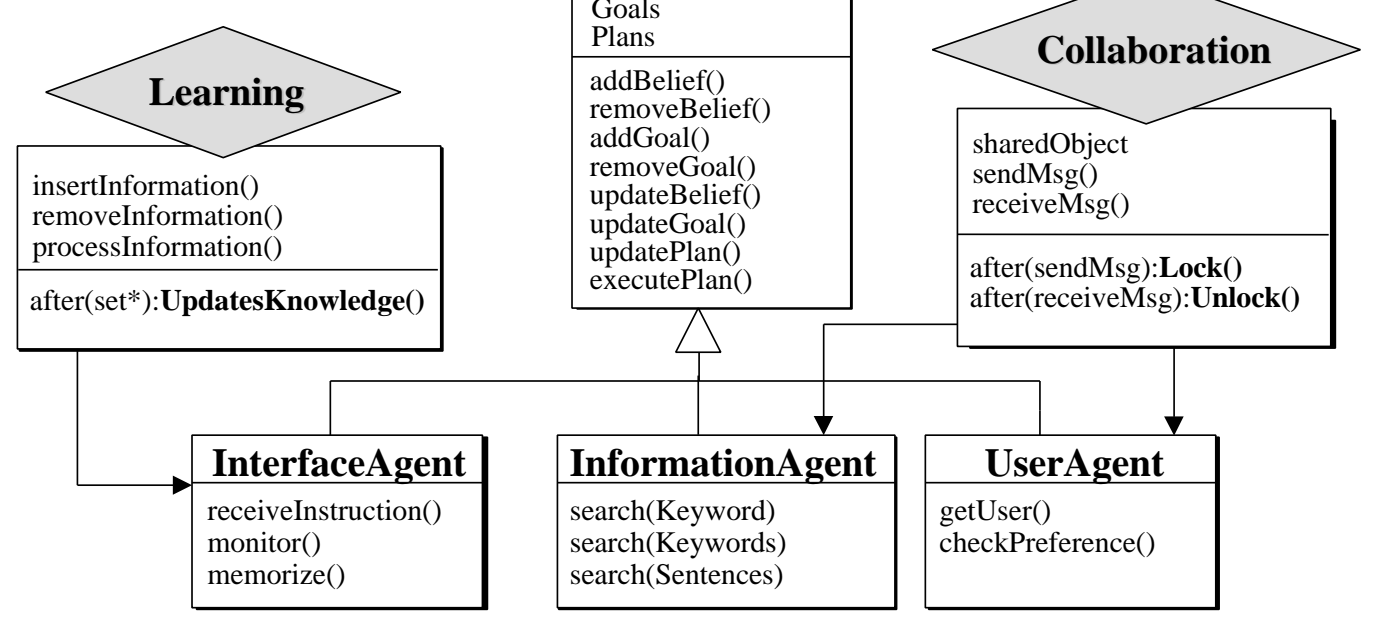

Figure 10. Additional Agency Aspects. 
Figure 11 illustrates this situation for the information agents of Portalware (Section 2.2). An information agent needs to play the caller and answerer roles in order to cooperate with other information agents in different contexts. . Thus, the Caller and Answerer role aspects are attached to the InformationAgent class. The Caller aspect provides an agent with the ability to send the search request to the answering agent as well receive the search result. Similarly, the Answerer aspect introduces the ability to receive the search request and to send the search result. The startsCaller() after advice is associated with receptions of searching methods (search(*)) and is responsible for sending the search request when the agent itself is not able to find the required information. This advice checks results of searching methods so that the caller is activated whenever the method result is null. Note that these roles are introduced in a way that is transparent and non-intrusive. Figure 11 pictures the application of our design approach for the Portalware agents. Note that the different types of software agents inherit the agent aspects attached to the Agent superclass. As a consequence, the information agent inherits agenthood features and only defines its specific services and agent aspects.

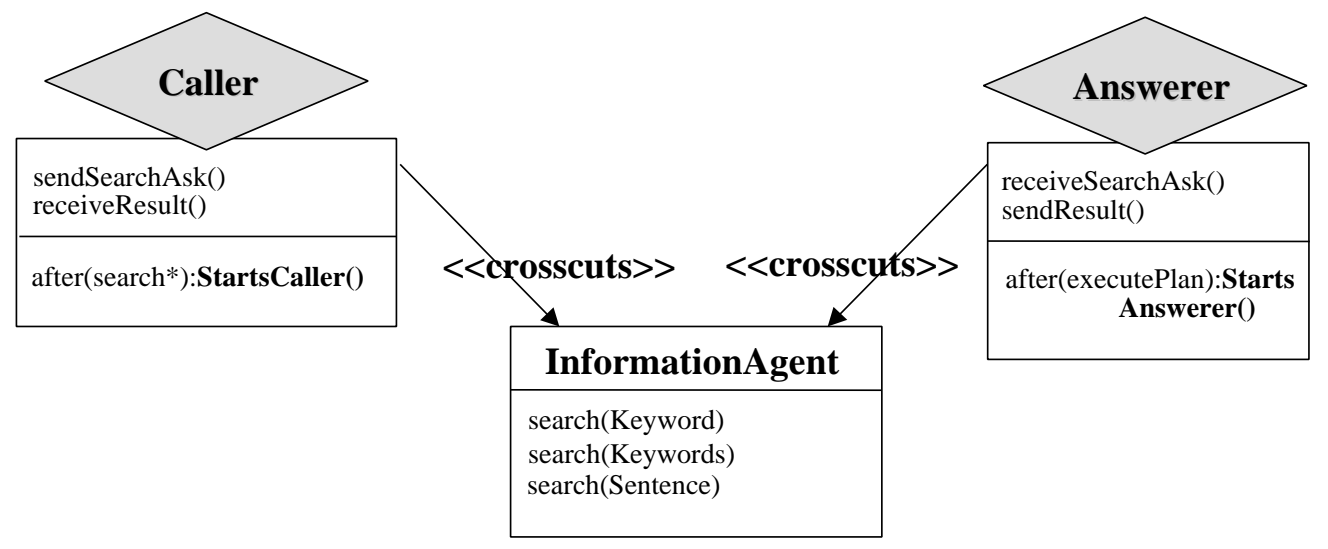

Figure 11. Role Aspects of Agents.

\subsection{Aspect Composition}

As we have stated previously (Section 2.1) agency properties are not orthogonal, they can overlap and interact with each other. As a result, there is a need for capturing the interactive and overlapping characteristics of the multiple agency aspects. Our method establishes relationship patterns which provide design rules that encompass the nonorthogonality of agency properties. To capture the interaction among agency aspects, we define an advice to each agency aspect at the same pointcut. For example, the Autonomy aspect interacts with the Interaction aspect in order to receive the incoming message and decide if the message should to be acepted. The Adaptation aspect interacts with the Autonomy aspect in order to adapt the agent state and behavior when a incoming message is acepted. As a consequence, these aspects implement different advices for the same pointcut that comprises receptions to calls to the receiveMsg() method. We use inheritance to capture the overlapping nature between the Interaction and the 
Collaboration aspects. Collaboration includes the interaction behavior and refines it to add the coordination protocol. So, the Collaboration aspect is a subaspect of the Interaction aspect (Figure 12).

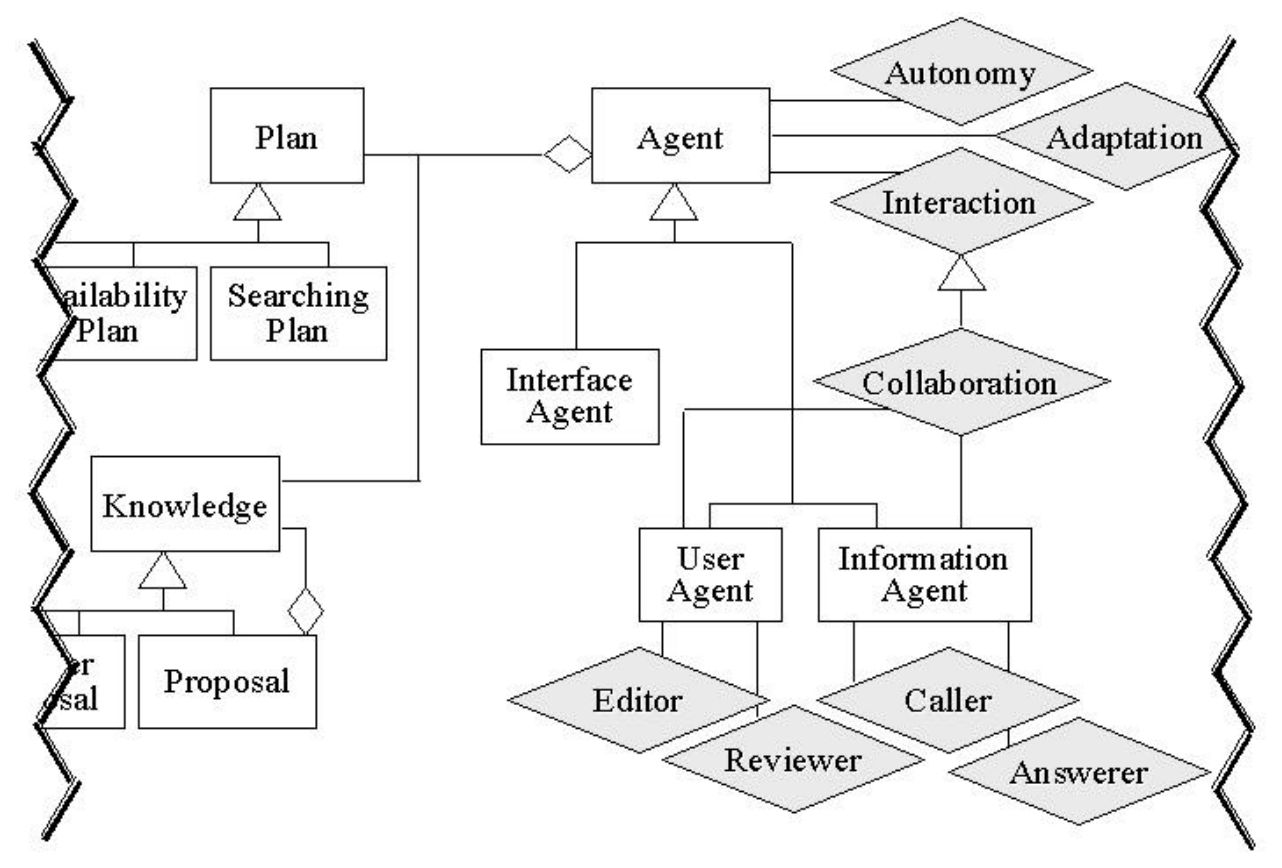

Figure 12. Portalware Agents and their Agency Aspects.

Figure 13 shows an interaction diagram for a basic call scenario between two information agents in Portalware. Weaver is the mechanism responsible for composing the multiple agency aspects. It directs the normal control flow to an advice when program execution is at a join point (Section 3.3). An information agent receives a message to search for information according to a specified keyword. The Interaction aspect receives this message through a sensor and updates the Inbox with the new message. The Autonomy aspect performs the decision-making process by invoking the decision plan. Thus, for every message that the agent receives, it may determine, based on its own goals and state, and the state of the ongoing conversation, whether to process the message and how to respond if it does. After the Autonomy aspect decides on processing the message, the Adaptation aspect adapts the agent's goals, since the agent must use a new search goal. When the goal is set, the Adaptation aspect seeks an appropriate agent plan for achieving this goal and adapts the agent's behavior for carrying out this search plan. The agent selects a plan on the basis of the current situation. During the plan execution, the agent's searching service is provided by the invocation of the search() method. Since the information agent is not able to find the specified keyword, the Caller aspect is activated in order to play the caller role. It calls the other information agent and asks for the required information. The Interaction aspect sends the message to the answerer information agent. After the message is sent, the Collaboration aspect carries out the coordination protocol so that the caller agent waits for the search result. Similarly, the agent receiveing the message plays its answerer role to receive the request and send the search result. It is worthwhile to highlight that advices and methods of dependent aspects are performed everytime after aspects on which they depend. 


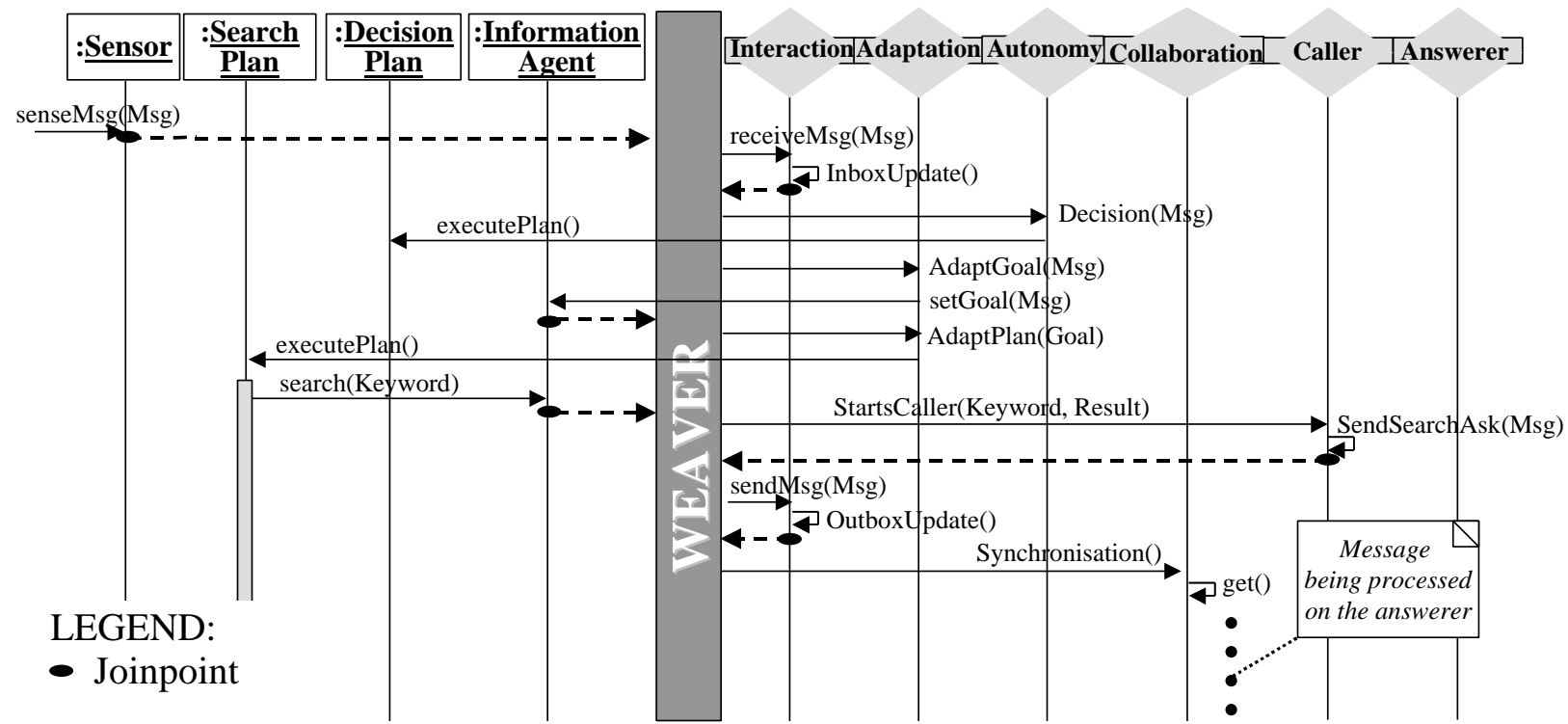

Figure 13. An Interaction Diagram for the Portwalware's Information Agents.

\subsection{Agent Evolution}

The behavior of software agents can evolve frequently to meet new application requirements. Suppose information agents do not need to cooperate with each other in order to find information. Instead, information agents are required to transport themselves from one environment in the network to another in order to achieve the searching goal. As a consequence, they do not need to have the caller and answerer roles, but must be mobile. In our model, this modification is done transparently, since agency aspects can be added to or removed from classes in a plug-and-play way. The Caller and Answerer aspects are detached from the InformationAgent class without requiring any invasive adaptation for the other agent's components (Figure 14). The remaining behavior of the agent is kept. However, it is necessary to associate the Mobility aspect, which introduces to the Agent class the ability to roam the network and gather information on behalf of its owner. This association process includes defining the end of executions of searching methods $\left(\operatorname{search}\left({ }^{*}\right)\right)$ as a pointcut. At runtime, when the execution of a search() method is finished, the weaver deviates the program control flow to the Mobility aspect. It takes the results of searching methods to check if the information agent was not able to find the information. Thus, if the method result is null, this aspect is responsible for migrating the agent to the other host in order to start a new search for the information. 

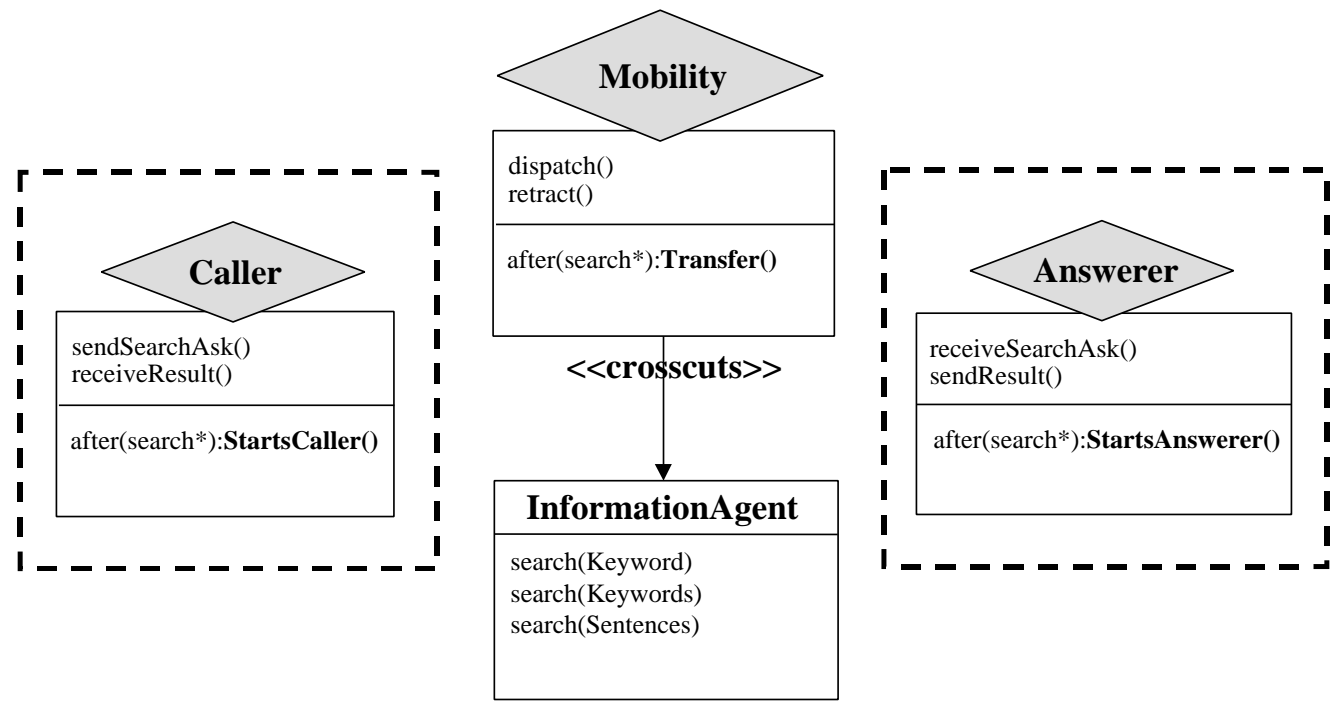

Figure 14. The Introduction of the Mobility Aspect.

\section{Implementation Issues}

Our aspect-based approach was implemented for the case study using AspectJ [24]. We have used AspectJ since, in our opinion, it is simpler and more integrated with the Java environment than the Hyper/J language. A more detailed comparison between these languages can be found in [50], where we survey the similarities and differences of these languages. The implementation consists of 91 classes, 5 agency aspects, and 5 role aspects. In order to implement the dependency relationship between these different aspects, we used the "dominates" construct of AspectJ. In addition, we have used two aspects, which implement an application's general aspects: exception handling and persistence. The persistence aspects are used for dealing with the persistence of an agent's state, while exception handling aspects are used to deal with both intra-agent exceptions and inter-agent exceptions.

In this sense, we have also used TSpaces/IBM [51], a blackboard architecture for network communication with database capabilities. TSpaces provides group communication services, database services and event notification services. It is implemented in the Java programming language and thus it automatically possesses network ubiquity through platform independence, as well as a standard type representation for all datatypes. In our prototype, messages captured by different collaborations were implemented as tuples and finally, agents communicated by posting such tuples to the blackboard. The only problem in using TSpaces and AspectJ together is that the persistence aspects crosscut classes of TSpaces and, as a result, we needed the source files of TSpaces during the weaving process.

In our case study, different instances of information agents should have varying characteristics. They may be collaborative or non-collaborative, they may be static or mobile, they may or may not learn. So, it is desirable to build personalized information agents to attach different aspects to distinct instances of information agents. Our proposed model supports this feature. 
Although we have established an internal architecture for the agents, which is in some way rooted in the BDI formalism [32, 33], different structures could also be used, such as the ones defined in specific implementation frameworks, such as $[18,19,20]$. The purpose of this paper is to stress a more generic design method instead of focusing on specific reasoning approaches. As a consequence, existing implementation frameworks can be used in conjunction with our method to produce different agent architectures. To implement the plans, we have used FIPA/KQML performatives. There are two Command patterns [23] introduced to accommodate all the FIPA/KQML speech act performatives. Their subclasses are named after the performatives, each subclass implements the functionality of the performative.

\section{Empirical Validation}

Experimental studies [52] are an effective way to supply empirical evidence that may improve our understanding about software engineering methods and techniques. A systematic evaluation has been carried out to validate the proposed method and demonstrate its usefulness, benefits and drawbacks in terms of relevant qualitative and quantitative criteria. The validation of the proposed method was conducted based on two distinct studies: a qualitative study and a quantitative study. In the first study, we have compared our aspect-oriented method with a patternoriented method based on qualitative criteria [26]. In the second study, we have developed a more controlled experiment [27] using Basili's GQM methodology [53], and a quality model with a metric suite for maintainability and reusability [54]. The second study was performed in order to confirm or refute the findings of the qualitative study. The following subsections present an overview of these experimental studies and the gathered results. The complete descriptions of these empirical studies and their outcomes are reported elsewhere [26, 27].

\subsection{Qualitative Study}

In this first study, we have carried out a comparative case study to evaluate the potential benefits and possible problems of applying our aspect-oriented method and a pattern-oriented method [26] to the design and implementation of Portalware (Section 2.2). This case study has been performed by two different teams working in parallel, with each team using one method. It has been structured into three phases: (i) initial system construction, (ii) subsequent modification due to new requirements (e.g., information agents become mobile), and (iii) reuse of existing features in new contexts (e.g., reuse of the Collaboration aspect in the context of User Agents). In phase 1, the two teams designed and implemented pattern-oriented and aspect-based solutions for Portalware based on the methods being investigated. Figure 2 shows a subset of the pattern-oriented design, while Figure 12 shows a subset of the aspect-oriented design of Portalware. On phases 2 and 3, both teams evolved the Portalware design and code, by modifying and reusing agency concerns, according to new requirements. The evaluation process considered 
qualitative criteria, regarding writeability, understandability, reusability, and maintainability as the key qualities. The comparative case study led to interesting results and insights concerning the overall benefits and usefulness of the aspect-based method, which are described in the following.

The aspect-oriented method supported writability better than the pattern-oriented method. With the aspect-based approach the subjects have written less code and furthermore, they were able to isolate agency concerns more naturally, and to compose them with little effort. The use of design patterns, with its demand on preplanning for change, requires the definition of a number of additional classes and methods with only trivial structure and behavior, e.g. abstract classes and explicit forwarding of messages to other objects or methods. This led to significant overhead for the software developer in terms of writability and also decreased understandability of the resulting code. For instance, the use of the Mediator and Role patterns requires the creation of additional classes, such as Property, CollaborativeAgent, CollaborationCore, and CollaborationRole (see Figure 2). These additional modules have not been created in the aspect-oriented design and code of Portalware.

A second important result is that the aspect-oriented method supported better reusability. Design patterns have no first-class representation at the implementation level. As a consequence, the implementation of a design pattern can therefore not be reused. Although its design is reused, the software developer is forced to implement the pattern many times. Unlike patterns, recent aspect-oriented programming languages [24, 55] provide first-class representation at the implementation level for agency aspects, supporting reuse both at the design and implementation levels. Moreover, our aspect-based approach supports better reuse as a side effect of AOSD; it supports the definition of implicit behavior composition at well-defined join points. For example, reusing the Collaboration property in the context of user agents required the association of the Collaboration aspect to UserAgent class, only depicting the join points of interest. In the pattern-based approach, some additional modifications were required to introduce this association as well as the explicit calls to methods defined in the interface of the Collaboration class.

The aspect-oriented method also supported better maintainability. Design for change, as prescribed by design patterns, sometimes imposed a not so simple structure to provide the required flexibility for evolution. During the maintenance phase, the introduction of the Mobility property was much simpler in the aspect-based design than in the pattern-based design. It required a new Mobility class that extended the Property class of the Mediator pattern [12]. The creation of this class was trivial but the maintenance process required intrusive modifications in the plans and actions using the mobility property of the agent. The subjects also had to detach all modules implementing the collaboration property from the InformationAgent class, which was a invasive and time-consuming task.

Finally, the aspect-oriented method supported better flexibility to accommodate distinct definitions for agenthood. Although we have presented a definition for agenthood (Section 2.1) that tries to identify the agency properties of 
software agents, this definition is not widely accepted and varies from researcher to researcher. This variation requires a development method flexible enough to encompass disciplined composition of agency aspects. Fortunately, our aspect-based approach can accommodate distinct definitions since agency aspects can be easily attached to and removed from the Agent class. An agent role prescribes the activity of the agents within a set of particular collaborations (Section 2.1). Moreover our design approach supports the introduction of multiple roles to the agent's core behavior in a disciplined manner. Last but not least, combining several design patterns in the same project was not a trivial task. It generated several alternative solutions to combine the patterns, where each of them presented advantages and limitations in relation to each other.

\begin{tabular}{|l|l|l|}
\hline \multicolumn{1}{|c|}{ Metrics } & \multicolumn{1}{|c|}{ Answered Questions } & \multicolumn{1}{c|}{ Principles } \\
\hline Vocabulary Size (VS) & How many components (classes/aspects) are in the design? & Size \\
\hline Lines of Code (LOC) & How many lines of code are there in the implementation? & Size \\
\hline Number of Attributes (NOA) & How many attributes are there in the components? & Size \\
\hline $\begin{array}{l}\text { Weighted Methods per } \\
\text { Component (WMC) }\end{array}$ & How many operations (methods/advices) are there? & Size \\
\hline $\begin{array}{l}\text { Coupling Between } \\
\text { Components (CBC) }\end{array}$ & How high is the coupling between components? & Coupling \\
\hline $\begin{array}{l}\text { Lack of Cohesion in Methods } \\
\text { (LCOM) }\end{array}$ & How high is the cohesion of the systems components? & Cohesion \\
\hline $\begin{array}{l}\text { Depth of Inheritance Tree } \\
\text { (DIT) }\end{array}$ & $\begin{array}{l}\text { How high is the coupling between components? } \\
\text { How high is the cohesion of the systems components? }\end{array}$ & $\begin{array}{l}\text { Coupling and } \\
\text { Cohesion }\end{array}$ \\
\hline $\begin{array}{l}\text { Concern Diffusion over } \\
\text { Components (CDC) }\end{array}$ & $\begin{array}{l}\text { How well are the agency concerns localized in terms of } \\
\text { components? }\end{array}$ & $\begin{array}{l}\text { Separation of } \\
\text { Concerns }\end{array}$ \\
\hline $\begin{array}{l}\text { Concern Diffusion over } \\
\text { Operations (CDO) }\end{array}$ & $\begin{array}{l}\text { How well are the agency concerns localized in terms of } \\
\text { operations? }\end{array}$ & $\begin{array}{l}\text { Separation of } \\
\text { Concerns }\end{array}$ \\
\hline $\begin{array}{l}\text { Concern Diffusions over } \\
\text { LOC (CDLOC) }\end{array}$ & How well are the agency concerns localized in termos of LOC? & $\begin{array}{l}\text { Separation of } \\
\text { Concerns }\end{array}$ \\
\hline
\end{tabular}

Table 2. Association between Metrics, GQM Questions and Principles

\subsection{Quantitative Study}

In the second study, we have replicated the comparative investigation of our aspect-oriented method and the same pattern-oriented method used in the qualitative study (Section 7.1). Unlike the qualitative evaluation, the overall goal of this empirical activity focused on the evaluation of the maintenance and reuse support of the investigated methods. Both methods were used in our empirical study to design and implement the same MAS. Moreover, this study was planed as a more controlled experiment [27] and a number of procedures were considered in order to minimize common problems in empirical software engineering [27, 52]. The subjects training and design/code standardization activities have been carried out in the beginning and end of the experiment, respectively. The application of the two methods was carried out by different developers. The evaluation of the two systems was conducted by using a quality model (Figure 15) and Basili's GQM methodology [53] based on which our metric suite was identified [54]. The GQM methodology was used to structure the experiment in terms of its goals, and to elicit the qualities, factors and 
principles investigated in this experiment. The GQM methodology is based upon the assumption that to gain a practical measure one must understand and specify the questions related to the software artifacts being measured.

Table 2 shows how the metric suite is related to the main questions and selected principles. The metric suite is composed of well-known metrics (such as VS and LOC), and adaptation of existing metrics (Chidamber's [56], for instance). Figure 15 illustrates our quality model and how it organizes the relationships between the metrics, principles, factors and qualities. The upper branches hold important high-level quality factors that we wanted to quantify. Each quality factor is related to traditional software engineering principles. The satisfaction of the principles is easier to measure than the factors. Then each principle is associated with a metric set, based on which actual measures are gathered. The data was partially gathered by the CASE tool Together 6.0 [57]. It supports some metrics: LOC, NOA, WMC (called WMPC2 in Together), CBC (called CBO in Together), LCOM (called LOCOM1 in Together) and DIT (called DOIH in Together).

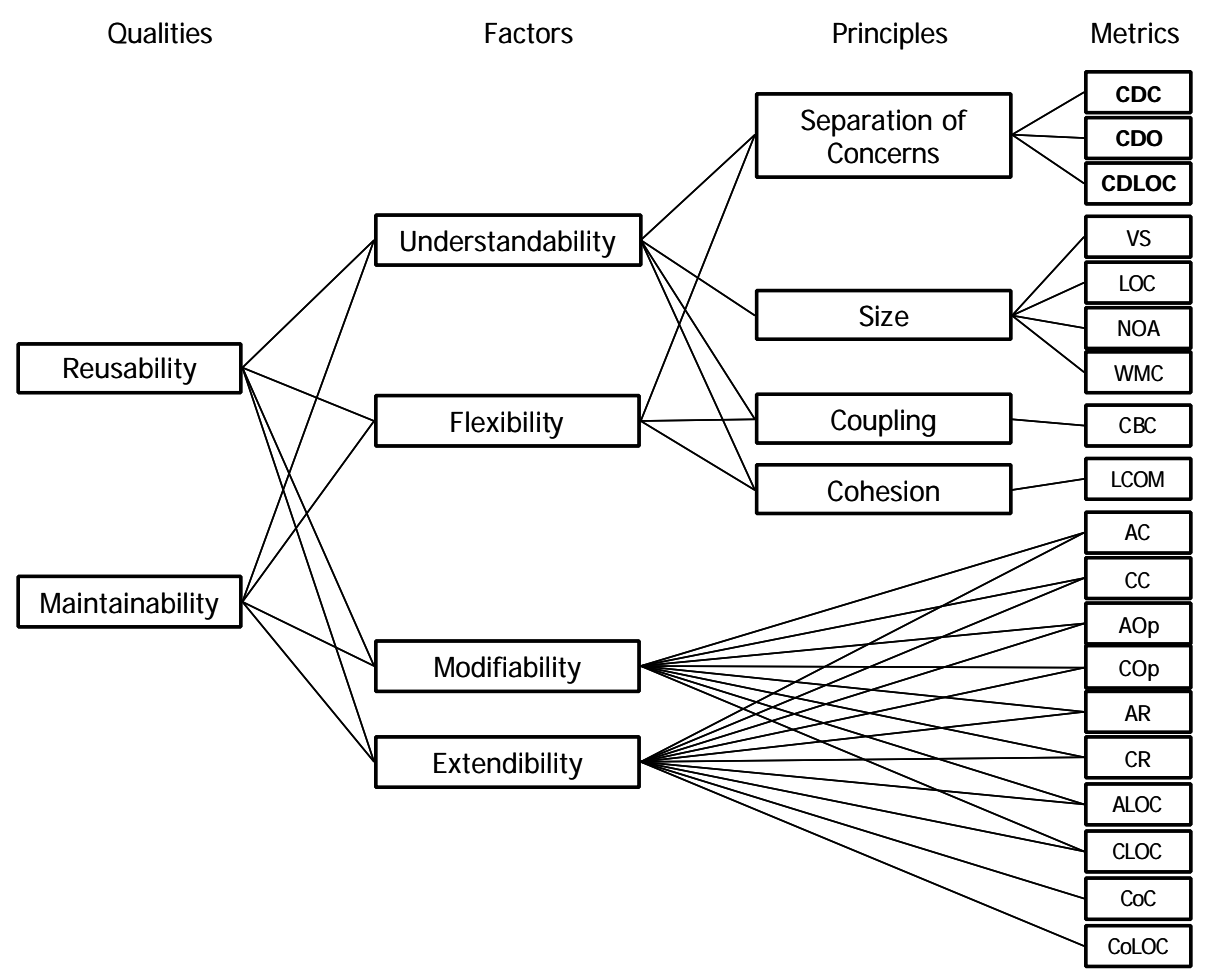

Figure 15. The Quality Model [27]

The metric suite was applied to provide software engineers with a better understanding of the interplay between agency concerns and objects in both investigated methods. The gathered results also provided a clear understanding of the strengths and weaknesses of the two investigated methods and their compatibility and divergences. In general, the results have shown that the aspect-oriented method provided better maintainability and reusability support than the pattern-oriented method. Figure 16 shows a sample of the results of some classes of the pattern-oriented MAS based on Together. Most results have confirmed the findings of the qualitative study. The gathered results have 
shown that the abstractions from the aspect-oriented approach allowed the construction of a MAS with improved separation of MAS concerns. Also, the use of this approach resulted in: (i) less lines of code, (ii) less components, (iii) lower component cohesion, and (iv) lower coupling between the components. In the following we discuss some of these central conclusions in this quantitative study. We refer to [27] for a complete description of the conclusions as well as the experiment organization.

1) The aspect-oriented method provided better support for separation of MAS concerns. It was found that the aspect-oriented mechanisms provide improved support to modularize agency concerns. This finding was supported by all separation of concerns metrics.

2) The aspect-oriented method produced a more concise MAS, in terms of lines of code, external vocabulary and internal vocabulary for the components. The use of design patterns leads to an increase in the number of classes dedicated to overcome the limitations of the composition mechanisms of object-oriented programming languages. This conclusion was supported by all size metrics, except the WMC metric. The use of the WMC metric detected the aspect-oriented method produced more complex operations, i.e advices, than the object-oriented method. It is because the modularization of some concerns in the aspects required the object contexts to be recaptured by means of pointcuts.

3) The pattern-oriented method produced more highly coupled components than the aspect-oriented method. This is a consequence of the lack of expressive power in the patterns to modularize MAS concerns. This result is supported by the $\mathrm{CBC}$ metric. The pattern-oriented method has lead to the abuse of the inheritance mechanism, which was responsible for establishing high inheritance couplings. This problem was detected by the DIT values.

4) The pattern-oriented method produced better results in terms of cohesion than the aspect-oriented method. The lack of cohesion in the aspects occurs because the aspect goal is to encapsulate behaviors, which act over different components. However, these behaviors cannot be directly related to each other, producing high LCOM values (i.e. low cohesion).

5) Both patterns and aspects were useful to minimize the misalignments between agent-oriented modeling and object-oriented design and implementation. The main difference between the aspect-oriented method and the patternoriented method is that the former explored aspects to represent roles and agent properties (such as adaptation, interaction, autonomy, collaboration, and so on), while the latter uses known patterns to represent those concerns. The main idea was the use of aspects to encapsulate agency properties and roles and separate them from the agent's basic functionality. The separation was very helpful to promote traceability among agent-oriented models and OO designs and code. However, the aspect notion provided better traceability since when an agency concern was added or 
removed during the agent-oriented modeling, it was more directly done in the aspect-oriented design and code. This finding was similar to the ones reported in [58].

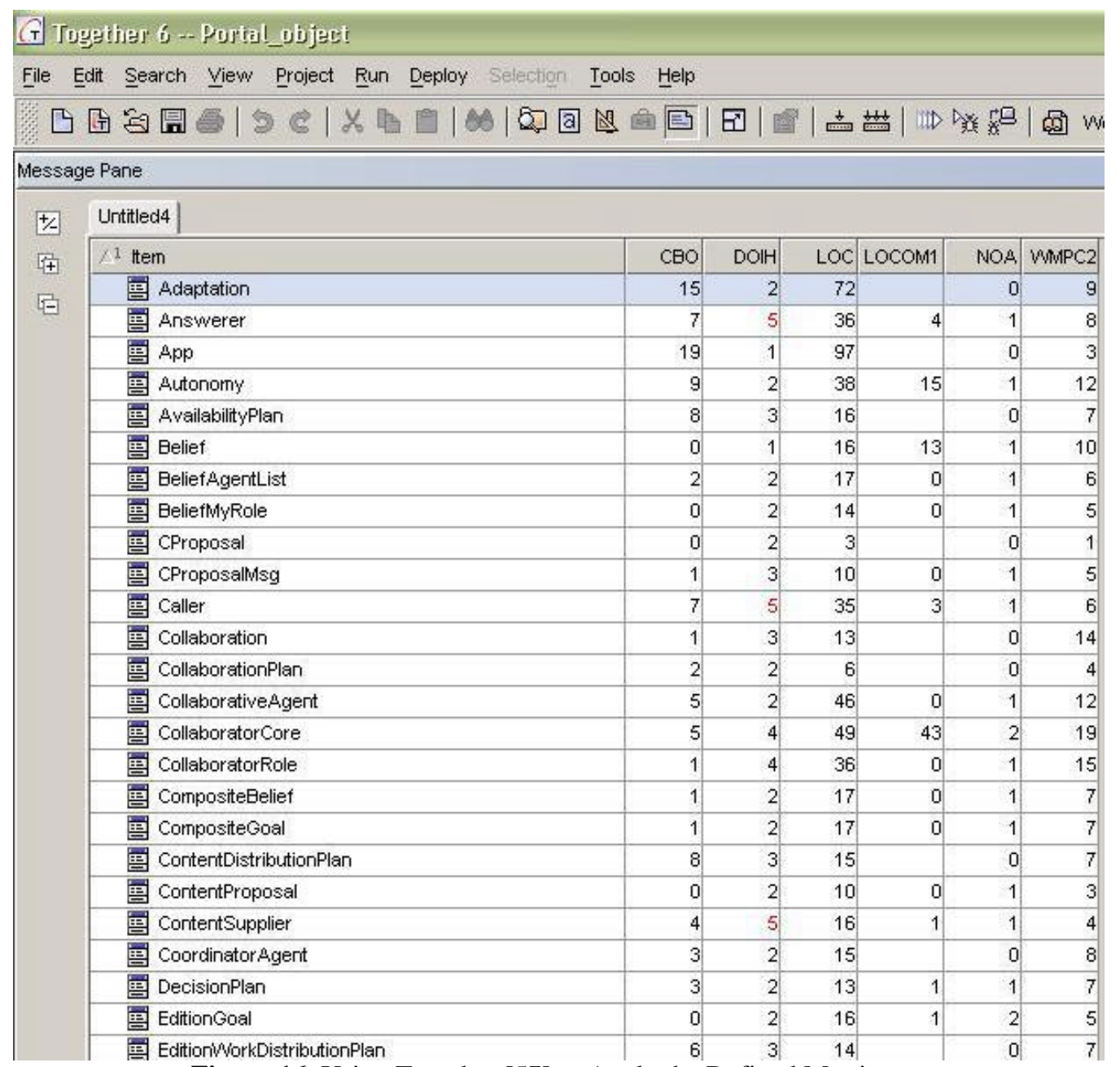

Figure 16. Using Together [57] to Apply the Defined Metrics

\section{Comparison with Related Work}

Research in agent-oriented software engineering has concentrated on high-level analysis and design methodologies $[3,7,44,46]$. These methodologies are too high level and do not indicate how to master the complexity of these concerns based on abstractions from the object paradigm. Even worse, these agent-oriented abstractions have not been satisfactorily identified in the light of object-orientation [7, 12]. As a consequence, it is difficult to understand the interplay between agents and objects in the MAS modeling and design. Moreover, it turns out the transition from high-level models to object-oriented programming is cumbersome and time-consuming. The MAS concerns are lost across the object-oriented code. From the implementation viewpoint, most research activities have focused on the definition of OO implementation frameworks (such as JADE [18]) to address specific agent properties. The lack of a 
unified modeling framework and principled methods to support the MAS concerns makes the MAS development and maintenance expensive and difficult.

Some few attempts to deal with agent complexity by using the object model have been proposed in the literature $[14,58]$. Kendall et al [14] proposes the layered agent architectural pattern, which separates different layers of an agent, such as sensory layer, action layer and so on. However, some aspects of agents, such as autonomy, cut across the different layers of this approach. We also believe evolution of this design is cumbersome since it is not trivial removing any of these layers; it requires the reconfiguration of the adjacent layers. This work does not present guidelines for evolving agent concerns in order to accommodate new agent aspects or remove existing ones. In fact, modeling the agency properties of an agent within the traditional object model is hard to do and introduces expressive limitations. In contrast, our approach allows the addition or removal of aspects of agents transparently (Section 4.7). Moreover, we have found that the use of design patterns for the agent domain introduces a number of problems [26]: (i) class explosion, (ii) need for preplanning, (iii) the application of the suitable design patterns is not trivial, and (iv) lack of expressive power. Finally, this proposal causes object shizophrenia - the agent state and behavior, which are intended to appear as a single object, are actually distributed over multiple objects.

Kendall et al [58] describes the application of aspect-oriented programming to implement role models. This approach is used to represent the different roles an agent can play during its lifetime. In fact, we have followed their guidelines to implement agent roles. However, their work does not deal with agents' agency properties, which we believe are the main source of agent complexity. In this sense, our paper presents a unified method for dealing with roles and other agency concerns, and their interrelationships.

A few works have presented aspect-based design solutions. In addition, since aspect-oriented programming is still in its infancy, little experience with employing this paradigm is currently available. To date, aspect-oriented programming has been used to implement generic aspects such as persistence, error detection/handling, logging, tracing, caching, and synchronization. However, each of these papers is generally dedicated to only one of these generic aspects. In this work, we provide an aspect-based approach which: (i) handles both agency-specific aspects as well as generic aspects (e.g. persistence), and (ii) encompasses a number of different aspects and their relationships.

\section{Conclusions and Future Work}

As the world moves rapidly toward the deployment of geographically and organizationally diverse computing systems, the technical difficulties associated with distributed, heterogeneous computing applications are becoming more apparent and placing new demands on software structuring techniques. The notion of agents is becoming increasingly popular in addressing these difficulties, and there is a need to understand how to integrate agent 
properties in a non-intrusive manner. In fact, it is not often easy to design software agents properly, as the developers of MASs have to take into account many agency properties at the same time. It is certainly not a trivial task since the complexity associated with the integration between agents and objects is not straighforward. The use of implementation frameworks and design patterns is not enough to deal with the problem.

This work discussed the problems in dealing with agency properties and capabilities as well as overviewed software engineering approaches to addressing these problems. So, we presented an aspect-based approach to make development of sophisticated agents simple enough to be practical. In fact, the main contribuition of this work is an implementation and design proposal that provides a unified framework for introducing complex software agents to the object model. Moreover, our proposed approach: (i) incorporates flexible facilities to build different kinds of software agents, (ii) encourages the handling of each of the agency aspects separately, (iii) provides explicit support for disciplined and transparent composition of agency properties and collaborative capabilities in complex software agents, and (iv) allows the production of agent-based software so that it is easy to understand, maintain and reuse. Our proposal explores the benefits of aspect-based software engineering for the incorporation of agency aspects in objectoriented systems. Since aspect-oriented programming is still in its infancy, little experience with employing this paradigm is currently available. In this sense, we have presented a substantial case study (Section 2.2) and two empirical studies, which we developed to validate our aspect-based approach. In addition, our method is currently being applied to develop a MAS for the urban traffic management domain and another one for the marketplace domain.

Design patterns [38] are important vehicles for constructing high-quality software. Architectural patterns define the basic structure of an architecture and of systems which implement that architecture; design patterns are more problem-oriented than architectural patterns, and are applied in later design stages. As presented in this paper, aspect-based design can be used to address the problems of dealing with agency properties. We are currently investigating a language of aspect-based design patterns for agent systems, which provide good design solutions for dealing with each of the agency aspects of agents. An architectural pattern should be proposed in order to specify a high-level description of the agent's organization in terms of its aspects and their interrelationships. Aspect-based design patterns can be used to provide solutions for each of the agency aspects of agents while following the overall structure of the proposed architectural pattern.

ACKNOWLEDGMENTS. This work has been supported partially by CNPq/Brazil under grant No. 141457/2000-7 for Alessandro Garcia, and also by FAPERJ under grant No. E-26/150.699/2002 for Alessandro. Alessandro and Carlos Lucena also are supported by the PRONEX Project under grant 7697102900, and by ESSMA under grant 
$552068 / 2002-0$ and by the art. $1^{\text {st }}$ of Decree number 3.800, of 04.20.2001. We would also like to thank Paulo Alencar,

Christina Chavez, Toacy Oliveira, Torsten Nelson and Arndt von Staa for the suggestions during this work.

\section{References}

1. N. Jennings and M. Wooldridge. "Agent-Oriented Software Engineering". Handbook of Agent Technology, J. Bradshaw (ed.). AAAI/MIT Press, 2000.

2. M. Wooldridge and P. Ciancarini. "Agent-Oriented Software Engineering: The State of the Art". Agent-Oriented Software Engineering, P. Ciancarini and M. Wooldridge (eds.). Springer-Verlag: LNAI 1957, Berlin, January 2001.

3. A. Garcia and C. Lucena. "Software Engineering for Large-Scale Multi-Agent Systems - SELMAS 2002". ACM Software Engineering Notes September 2002; 27(5): 82-88.

4. A. Pace, F. Trilnik, M. Campo. "Assisting the Development of Aspect-based MAS using the SmartWeaver Approach". Software Engineering for Large-Scale Multi-Agent Systems, A. Garcia, C. Lucena, J. Castro, A. Omicini, F. Zambonelli (eds.). Springer-Verlag: LNCS 2603, Berlin, April 2003.

5. D. Parnas. "On the Criteria To Be Used in Decomposing Systems into Modules". Communications of the ACM December 1972; 15(12): 1053 - 1058.

6. A. Garcia, C. Lucena, J. Castro, A. Omicini, F. Zambonelli (eds.). Software Engineering for Large-Scale MultiAgent Systems. Springer-Verlag: LNCS 2603, Berlin, April 2003.

7. J. Debenham, B. Henderson-Sellers, N. Jennings, J. Odell (eds). Proceedings of the OOPSLA 2002 Workshop on Agent-Oriented Methodologies. COTAR, 2002.

8. M. Elammari and W. Lalonde. "An Agent-Oriented Methodology: High-Level and Intermediate Models". Proceedings of the Workshop on Agent-Oriented Information Systems (AOIS 1999), Heidelberg, Germany, June 1999.

9. M. Wooldridge, N. Jennings, D. Kinny. "The Gaia Methodology for Agent-Oriented Analysis and Design". Autonomous Agents and Multi-Agent Systems 2000; 3(3): 285 - 312.

10. E. Letier and A. van Lamsweerde. "Agent-based Tactics for Goal-Oriented Requirements Elaboration". Proceedings of 24th International Conference on Software Engineering, Toronto, Canada, May 2001.

11. G. Wagner. "Agent-Object-Relationship Modeling”. Proceedings of the 2nd International Symposium: From Agent Theory to Agent Implementation, April 2000.

12. V. Silva, A. Garcia, A. Brandão, C. Chavez, C. Lucena, P. Alencar. "Taming Agents and Objects in Software Engineering". Software Engineering for Large-Scale Multi-Agent Systems, A. Garcia, C. Lucena, J. Castro, A. Omicini, F. Zambonelli (eds.). Springer-Verlag: LNCS 2603, Berlin, April 2003.

13. J. Bradshaw, S. Dutfield, P. Benoit, J. Woolley. "KaoS: Toward an Industrial-Strength Generic Agent Architecture". Software Agents, J. M. Bradshaw (ed.). AAAI/MIT Press: Cambridge, MA, 1996.

14. E. Kendall, P. Krishna, C. Pathak, C. Suresh. "A Framework for Agent Systems". Implementing Application Frameworks - Object-Oriented Frameworks at Work, M. Fayad et al. (eds.). John Wiley \& Sons: 1999.

15. D. Lange and M. Oshima. Programming and Developing Java Mobile Agents with Aglets. Addison-Wesley: August 1998.

16. G. Kiczales, J. Lamping, A. Mendhekar, C. Maeda, C. Lopes, J. Loingtier, J. Irwin. "Aspect-Oriented Programming". Proceedings of the European Conference on Object-Oriented Programming (ECOOP'97), Finland, June 1997. Springer-Verlag: LNCS 1241, 1997.

17. P. Tarr, H. Ossher, W. Harrison, S. Sutton. "N Degrees of Separation: Multi-Dimensional Separation of Concerns." Proceedings of the 21st International Conference on Software Engineering (ICSE'99), May 1999.

18. F. Bellifemine, A. Poggi, G. Rimassi. "JADE: A FIPA-Compliant Agent Framework." Proceedings of the Practical Applications of Intelligent Agents and Multi-Agents, April 1999; 97-108.

19. H. Nwana, D. Ndumu, L. Lee, J. Collis. "ZEUS: An advanced Toolkit for Engineering Distributed Multi-Agent Systems". Applied Artificial Intelligence Journal 1999, 13(1):129-186.

20. K. Sycara, M. Paolucci, M. van Velsen, J. Giampapa. "The RETSINA MAS Infrastructure.” Autonomous Agents and Multi-Agent Systems July 2003, 7(1-2).

21. Foundation for Intelligent Physical Agents, Agent Communication Technical Committee. "Agent Communication Language". FIPA'99 Draft Specification, 1999. http://www.fipa.org. 
22. T. Finin, R. Fritzson, D. McKay, R. McEntire. "KQML as an Agent Communication Language". Proceedings of the Third International Conference on Information and Knowledge Management, 1994; 456-463.

23. A. Garcia, M. Cortés, C. Lucena. "A Web Environment for the Development and Maintenance of E-Commerce Portals Based on Groupware Approach". Proceedings of the 2001 Information Resources Management Association International Conference (IRMA'O1), Toronto, Canada, May 2001; 722-724.

24. G. Kiczales et al. "An Overview of AspectJ". Proceedings of the European Conference on Object-Oriented Programming (ECOOP'01), Budapest, Hungary, 2001.

25. J. Gosling, B. Joy, G. Steele. The Java Language Specification. Addison-Wesley, 1996.

26. A. Garcia, V. Silva, C. Chavez, C. Lucena. "Engineering Multi-Agent Systems with Aspects and Patterns". Journal of the Brazilian Computer Society July 2002, 8(1):57-72.

27. A. Garcia, C. Sant'Anna, C. Chavez, V. Silva, C. Lucena, A. von Staa. "Agents and Objects: An Empirical Study in the Design and Implementation of Multi-Agent Systems". Proceedings of the Workshop on Software Engineering for Large-Scale Multi-Agent Systems (SELMAS'03) at ICSE'03, Portland, USA, May 2003; 11-21.

28. Object Management Group - Agent Platform Special Interest Group. Agent Technology - Green Paper. Version 1.0, September 2000.

29. Z. Guessoum and J. Briot. "From Active Objects to Autonomous Agents". IEEE Concurrency 1999 (Special Series on Actors and Agents), 7(3): 68-76.

30. J. Bradshaw. “An Introduction to Software Agents”. Software Agents, J. Bradshaw (ed.). AAAI /MIT Press, 1997.

31. Y. Shoham. “Agent-Oriented Programming”. Artificial Intelligence 1993, 60(1):51-92.

32. A. Rao and M. Georgeff. "Modelling Rational Agents within a BDI Architecture". Proceedings of the 2nd International Conference on Principles of Knowledge Representation and Reasoning, Cambridge, MA, 1991; 473-484.

33. A. Rao and M. Georgeff. "BDI Agents: From Theory to Practice". Proceedings of the 1st International Conference on Multi-Agent Systems (ICMAS-95), San Francisco, 1995; 312-319.

34. H. Nwana. "Software Agents: An Overview". Knowledge Engineering Review September 1996, 11(3): 1-40.

35. P. Ripper, M. Fontoura, A. Neto, C. Lucena. "V-Market: A Framework for e-Commerce Agent Systems." World Wide Web 2000, Baltzer Science Publishers, 3(1): 43-52.

36. SoC+Agents Group. Separation of Concerns and Multi-Agent Systems. URL: http://www.teccomm.les.inf.pucrio.br/SoCagents/

37. G. Booch and J. Rumbaugh. Unified Modeling Language - User Guide. Addison-Wesley, 1999.

38. E. Gamma, R. Helm, R. Johnson, J. Vlissides. Design Patterns: Elements of Reusable Object-Oriented Software. Addison-Wesley, Reading, MA, 1995.

39. D. Bäumer et al. "Role Object". Proceedings of the 1997 Conference on Pattern Languages of Programs (PLoP '97), 1997.

40. O. Silva, A. Garcia, C. Lucena. "The Reflective Blackboard Pattern: Architecting Large-Scale Multi-Agent Systems". Software Engineering for Large-Scale Multi-Agent Systems, A. Garcia, C. Lucena, J. Castro, A. Omicini, F. Zambonelli (eds.). Springer-Verlag: LNCS 2603, Berlin, April 2003.

41. A. Finkelstein, J. Kramer, B. Nuseibeh, L. Finkelstein, M. Goedicke. "Viewpoints: a Framework for Integrating Multiple Perspectives in System Development". International Journal of Software Engineering and Knowledge Engineering, 2(1):31-37; 1992.

42. K. Kang et al. "Feature-Oriented Domain Analysis (FODA): Feasibility Study". Technical Report CMU/SEI-90TR-21, Pittsburgh, PA: Software Engineering Institute, Carnegie Mellon University, 1990.

43. W. Harrison and J. Ossher. "Subject-Oriented Programming: A Critique of Pure Objects". Proceedings of the ACM Conference on Object-Oriented Programming, Systems, Languages, and Applications (OOPSLA'93), ACM Press, 1993; 411-428.

44. C. Iglesias, M. Garrijo, J. Gonzalez. "A Survey of Agent-Oriented Methodologies." Proceedings of the 5th International Workshop on Intelligent Agents: Agent Theories, Architectures, and Languages (ATAL-98), Paris, France, July 1998; 317-330.

45. M. Wooldridge, N. Jennings. "Agent Theories, Architectures, and Language: A Survey". Proceedings of the ECAI-94 Workshop on Agent Theories, Architectures, Languages, M. Wooldridge and N. Jennings (eds.). SpringVerlag: Berlin, 1994; 1-39.

46. A. Karageorgos, S. Thompson, N. Mehandjiev. "Semi-Automatic Design of Agent Organisations". Proceedings of the ACM Symposium on Applied Computing, Madrid, Spain, March 2002; 306-313

47. M. Fayad et al. Implementing Application Frameworks. First Edition, John Wiley \& Sons, 1999.

48. FIPA: Foundation for Intelligent Physical Agents. http://www.fipa.org. 
49. J. Odell, H. Parunak, B. Bauer. "Extending UML for Agents". Proceedings of the Agent-Oriented Information Systems Workshop at the 17th National Conference on Artificial Intelligence, J. Odell, H. Parunak, B. Bauer (eds.), 2000.

50. C. Chavez, A. Garcia, C. Lucena. "Some Insights on the Use of AspectJ and Hyper/J". Proceedings of the Tutorial and Workshop on Aspect-Oriented Programming and Separation of Concerns, Lancaster, UK, August 2001.

51. T. Lehman, S. McLaughry, P. Wyckoff. "TSpaces: The Next Wave”. Hawaii International Conference on System Sciences (HICSS-32), January 1999.

52. V. Basili, R. Selby, D. Hutchen. "Experimentation in Software Engineering". IEEE Transactions on Software Engineering 1986, 12(7):733-743.

53. V. Basili, G. Caldiera, H. Rombach. "The Goal Question Metric Approach”. Encyclopedia of Software Engineering September 1994, 2(9): 528-532. John Wiley \& Sons, 1994

54. C. Sant'Anna, A. Garcia, C. Chavez, C. Lucena, A. von Staa. "On the Reuse and Maintenance of Aspect-Oriented Software: An Assessment Framework." Proceedings of 17th Brazilian Symposium on Software Engineering, Manaus, Brazil, October 2003.

55. P. Tarr and H. Ossher. “Hyper/J User and Installation Manual”. http://www.alphaworks.ibm.com/tech/hyperj

56. S. Chidamber, C. Kemerer. “A Metrics Suite for Object Oriented Design”. IEEE Transactions on Software Engineering June 1994, 20(6): 476-493.

57. Borland Together ControlCenter 6.0. http://www.togethersoft.com/

58. E. Kendall. "Role Model Designs and Implementations with Aspect-oriented Programming". Proceedings of the ACM Conference on Object-Oriented Programming, Systems, Languages, and Applications (OOPSLA'99), ACM Press, 1999; 353-369.

59. C. Chavez, C. Lucena. "Design-level Support for Aspect-oriented Software Development". Proceedings of the Workshop on Advanced Separation of Concerns in Object-Oriented Systems (ASoC) at OOPSLA'2001, Tampa Bay, Florida, USA, October 14, 2001. 